\title{
QUAND UNE VACHE EST-ELLE RASSASIÉE? \\ PAR
}

\section{André VOISIN}

Agriculteur, Président de l'Union des Coopératives laitières de la Seine-Inférieure.

\section{INTRODUCTION}

\section{Le cultivateur et les tables d'alimentation}

Malgré tous les efforts entrepris dans tous les pays pour vulgariser l'emploi par le cultivateur des tables d'alimentation, en particulier dans le cas des vaches à lait, on peut dire que le succès a été très faible et très limité. Que ce soit dans les revues ou dans des conversations entre dirigeants agricoles et savants, on lit et on entend des plaintes renouvelées sur la répugnance des cultivateurs, même éclairés et instruits, à régler et calculer les rations des vaches à lait d'après les tables d'alimentation. Pour éviter au cultivateur tout calcul, on a même dressé des rations types où, pour des vaches de différents rendements, on indique des rations diverses réalisées avec les aliments que le cultivateur pourra avoir à sa disposition. Or, malgré cela, le cultivateur continue à très peu utiliser ces rations-types.

Quelle peut être la cause de cette répugnance du cultivateur à employer les tables d'alimentation et les rations-types? Une réponse simple consiste à dire : "Le cultivateur est un rétrograde et un ignorant ". Or, s'il existe des cultivateurs rétrogrades et ignorants comme il existe du reste des individus de cette espèce dans toutes les professions, il y a une catéorie de cultivateurs auxquels on ne peut certainement pas appliquer ces épithètes: ce sont les éleveurs, hommes qui par un travail patient, nécessitant un esprit d'observation pénétrant et continuellement en éveil, ont réussi à améliorer nos grandes races laitières et à créer ces championnes magnifiques qui sont l'honneur de ces races. Or, ces éleveurs-là sont parfaitement capables de comprendre les tables d'alimentation et encore mieux les rations-types. Il faut cependant avoir la franchise de dire qu'ils ne tiennent guère compte des tables d'alimentation pour la nourriture de leurs vaches. Ils procèdent par tâtonnement et voient les rations qui font " monter " ots " tomber " la production de leurs vaches. 
Ayons done le courage et la franchise de dire qu'il y a quelque chose qui ne "colle " pas dans nos tables d'alimentation. J'ai eu chez moi comme stagiaires des fils de nos grands éleveurs normands; et je leur ai expliqué le maniement des tables. Par la suite, quand ils retournèrent chez eux et que je leur demandai quelle application ils faisaient de ces tables, ils me firent comprendre d'un air embarrassé, que ces tables les guidaient " un peu ", leur donnaient des indications "utiles ", leur permettaient de mieux "équilibrer " leurs rations. Mais, il n'était pas question d'une application systématique et suivie. Comme j'avais pu apprécier la valeur de ces jeunes gens, j'étais bien obligé d'admettre qu'il y avait de sérieuses difficultés d'application, lesquelles, à dire vrai, je connaissais bien par mon expérience personnelle.

Je ne ferai pas part de toutes les expériences personnelles que $j$ 'ai subies avec peine, et me contenterai de rapporter la suivante:

En hiver, je donne à mes vaches une certaine ration de base de concentré adaptée le mieux possible (du moins, je le pense), à la production de lait de la vache. On donne un peu de fourrage sec, et la vache mange tout ce qu'elle veut d'ensilage, tout en le limitant de manière à éviter qu'elle laisse de son concentré. Cet ensilage est constitué surtout par des queues de betteraves mélangées avec un peu de pulpe. On donne également un peu de jeune luzerne ensilée.

Par suite de circonstances matérielles, on dut retarder en novembre l'ouverture du silo à queues de betteraves. Je ne m'inquiétais pas et faisais donner uniquement de l'ensilage de luzerne coupée au début de floraison, que cette année-là je possédais en quantités importantes. A dire vrai, j'étais même fort heureux de l'emploi de cette nourriture, tellement plus riche en protéine que les queues de betteraves. J'estimais que pour I ooo $\mathrm{g}$ de matière sèche on avait les richesses suivantes:

\begin{tabular}{|c|c|c|}
\hline !Pour I $000 \mathrm{~g}$ de matière sèche & $\begin{array}{l}\text { Protéine digestibl } \\
\mathrm{g}\end{array}$ & $\begin{array}{c}\text { Inités Amidon } \\
\text { g }\end{array}$ \\
\hline $\begin{array}{l}\text { Tourteau de lin } \ldots \ldots \ldots \ldots \ldots \ldots \ldots \\
\text { Silage luzerne (début floraison) } \ldots \ldots \ldots \ldots \\
\text { Silage queues de betteraves . . . . . . }\end{array}$ & $\begin{array}{r}276 \\
{ }^{1} 30 \\
77\end{array}$ & $\begin{array}{l}780 \\
465 \\
467\end{array}$ \\
\hline
\end{tabular}

Nous avions donc là un ensilage beaucoup plus riche en protéines que celui habituellement employé et qui se rapprochait des tourteaux. Cet apport des protéines rares et désirées ne parut pas avoir d'effets heureux ; la production du troupeau de quarante vaches était loin d'atteindre celle des années précédentes. De plus, on constata que les vaches mangeaient des quantités beaucoup plus faibles d'ensilage de luzerne que de queues de betteraves.

Devant cette expérience malheureuse, dès que je pus ouvrir mon silo à queues de betteraves, je décidais pour mieux voir la différence de totalement supprimer pour quelques jours l'ensilage de luzerne et de le remplacer par 
l'ensilage de queues de betteraves (qui contenait un peu de pulpes). En moins de quinze jours, la production globale du troupeau avait monté de $20 \%$.

Autrement dit, une nourriture moitié moins riche en protéines et ayant une valeur amidon équivalente augmentait le rendement. Il y avait vraiment de quoi mettre au feu toutes les tables d'alimentation.

Il est extrêmement difficile pour un cultivateur de faire des mesures précises sur tout un troupeau. Je m'efforçais cependant de le faire sur un groupe de vaches dont la production moyenne était de 20 litres environ. Ce groupe était tombé au-dessous de 20 litres avec le silage de luzerne et au contraire avait eu tendance à remonter au-dessus de 20 litres avec les queues de betteraves. De plus, on avait observé que les vaches de ce groupe absorbaient en moyenne $30 \mathrm{~kg}$ de silage de luzerne alors qu'elles absorbaient au moins $70 \mathrm{~kg}$ de silage de queues de betteraves.

La ration de base de ces vaches avec les deux ensilages était en moyenne de :

$2 \mathrm{~kg}$ d'avoine.

$2 \mathrm{~kg}$ de tourteau de lin.

$3 \mathrm{~kg}$ de fourrage de trèfle de bonne qualité.

Cette ration avait donc les caractéristiques suivantes:

\begin{tabular}{|c|c|c|c|c|}
\hline & $\begin{array}{l}\text { Matière } \\
\text { séche } \\
\text { gr }\end{array}$ & $\begin{array}{l}\text { Protéines } \\
\text { digestibles } \\
\quad \mathrm{g}\end{array}$ & $\begin{array}{l}\text { Valeur } \\
\text { Amidon } \\
\quad \mathrm{g}\end{array}$ & $\begin{array}{c}\text { Ballast } \\
\mathrm{g}\end{array}$ \\
\hline $\begin{array}{l}2 \mathrm{~kg} \text { d'avoine } \ldots \ldots \ldots \ldots \ldots \ldots \ldots \ldots \\
2 \mathrm{~kg} \text { de lin } \ldots \ldots \ldots \ldots \ldots \ldots \ldots \ldots \\
3 \mathrm{~kg} \text { de fourrage de trète (bon) } \ldots \ldots \ldots\end{array}$ & $\begin{array}{l}1720 \\
1780 \\
2580\end{array}$ & $\begin{array}{l}174 \\
492 \\
201\end{array}$ & $\begin{aligned} \text { I } 26.4 \\
\text { I } 388 \\
975\end{aligned}$ & $\begin{array}{l}428 \\
356 \\
930\end{array}$ \\
\hline Total. ...... & 6080 & 867 & 3627 & 1714 \\
\hline
\end{tabular}

\section{RÉFLEXIONS SUR LE RASSASIEMENT}

Le fait que les vaches absorbaient $70 \mathrm{~kg}$ de silage de queues de betteraves contre une trentaine de kilos de silage de luzerne me semblait amener la conclusion logique :

"L'ensilage de luzerne rassasie la vache plus vite que l'ensilage de queues de betteraves. "

La question qui s'ensuivait immédiatement était :

"Quel est le facteur qui dans un aliment détermine le rassasiement d'une vache?"

La théorie la plus courante de nos ouvrages d'alimentation français ou étrangers est que ce rassasiement est déterminé par la quantité de matière sèche absorbée, laquelle est de I2 à $I_{5} \mathrm{~kg}$ pour une vache de $500 \mathrm{~kg}$.

Voyons donc les quantités de matière sèche de nos deux silages. 
Si nous supposons qu'une vache de $500 \mathrm{~kg}$ peut absorber $\mathrm{I}_{4} \mathrm{~kg}$ de matière sèche, la ration fixe de base contenant $6080 \mathrm{gr}$ de matière sèche, il reste une

\begin{tabular}{c|c}
\hline Pour 1 ooo g d'aliment brut, on $\mathbf{a}:$ & $\begin{array}{c}\text { Matière sèche } \\
\mathbf{g}\end{array}$ \\
\hline Silage luzerne (début floraison) $\ldots \ldots \ldots \ldots \ldots \ldots$ & $\begin{array}{c}270 \\
200\end{array}$ \\
Silage queues de betteraves............. & \\
\hline
\end{tabular}

absorption disponible de :

$\mathrm{I}_{4} 000-6080=7920 \mathrm{gr}$ de matière sèche,

A cette quantité de matière sèche correspondent les quantités d'aliment brut et de substances nutritives ci-dessous :

\begin{tabular}{|c|c|c|c|}
\hline Pour $7920 \mathrm{~g}$ de matière sèche, on a : & $\begin{array}{c}\text { Aliment } \\
\text { brut } \\
g\end{array}$ & $\begin{array}{c}\text { Protéines } \\
\text { digestibles } \\
g\end{array}$ & $\begin{array}{c}\text { Unités } \\
\text { Amidon } \\
g\end{array}$ \\
\hline $\begin{array}{l}\text { Silage luzerne (début floraison) } \ldots \ldots \ldots \ldots \ldots \ldots \\
\text { Silage queues de betteraves. . . . . . . . . }\end{array}$ & $\begin{array}{l}29300 \\
39600\end{array}$ & $\begin{array}{r}\text { I } 027 \\
594\end{array}$ & $\begin{array}{l}3700 \\
3680\end{array}$ \\
\hline
\end{tabular}

La théorie du rassasiement par la matière sèche explique donc que la vache mange un poids plus élevé de silage de queues de betteraves que de silage de luzerne, tout en indiquant un poids de silage de queues de betteraves inférieur à celui absorbé en réalité.

Mais, au point de vue des éléments nutritifs, le silage de luzerne apporte la même quantité d'unités amidon et le double de protéines, vis-à-vis du silage de queues de betteraves. Il semble donc que la théorie du rassasiement par la matière sèche n'explique en rien la chute de production due à l'emploi de silage de luzerne.

Une autre théorie du rassasiement est celle du ballast, théorie développée par le savant allemand LEHMANN. Celui-ci appelle ballast la partie non digestible des matjères organiques de l'aliment. I1 considère que c'est cette partie non digestible seule qui (non absorbée par l'organisme comme la partie digestible), détermine le remplissage et par suite la limite d'absorption du système digestif. LEHMANN a admis que la quantité maxima de ballast que pouvait absorber une vache de $500 \mathrm{~kg}$ était de $4,3 \mathrm{~kg}$. Revoyons donc les caractéristiques de nos deux silages, en particulier en ce qui concerne le ballast. Nous avons :

\begin{tabular}{|c|c|c|c|c|}
\hline Pour r $000 \underset{n}{\mathrm{~g}}$ aliment brut, on a : & $\begin{array}{c}\text { Maticre } \\
\text { stche } \\
\text { :x }\end{array}$ & $\begin{array}{c}\text { Protéines } \\
\text { digestibles } \\
\qquad\end{array}$ & $\begin{array}{c}\text { Unités } \\
\text { Amidon } \\
\mathrm{g}\end{array}$ & liallast \\
\hline $\begin{array}{l}\text { Silage luzerne (floraison) } \ldots \ldots \ldots \ldots \ldots \ldots \ldots \\
\text { Silage queues de betteraves } \ldots \ldots \ldots \ldots \ldots \ldots\end{array}$ & $\begin{array}{l}270 \\
200\end{array}$ & $\begin{array}{l}35 \\
15\end{array}$ & $\begin{array}{r}126 \\
93\end{array}$ & $\begin{array}{l}92 \\
36\end{array}$ \\
\hline
\end{tabular}


Comme notre ration fixe de base contenait I 7 I 4 gr de ballast, nous pouvons encore faire absorber:

$4300-$ I $7 \mathrm{r}_{4}=25^{86} \mathrm{gr}$ de ballast.

A cette quantité de ballast correspondent les éléments suivants :

\begin{tabular}{|c|c|c|c|c|}
\hline & $\begin{array}{c}\text { Aliment } \\
\text { brut } \\
g\end{array}$ & $\begin{array}{c}\text { Matière } \\
\text { sèche } \\
g\end{array}$ & $\begin{array}{c}\text { Protéines } \\
\text { digestibles } \\
g\end{array}$ & $\begin{array}{c}\text { Unités } \\
\text { Amidon } \\
\qquad\end{array}$ \\
\hline 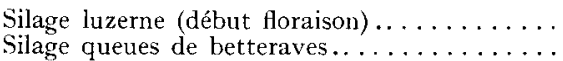 & 29200 & 7880 & I 020 & 3670 \\
\hline Silage queues de betteraves $\ldots \ldots \ldots \ldots \ldots \ldots$ & 74600 & 14900 & I I 20 & 6940 \\
\hline
\end{tabular}

La première constatation qui saute aux yeux, c'est que la théorie du ballast indique la possibilité d'absorption de quantités de silage correspondant à peu près à celles effectivement absorbées, ce qui ne laisse pas d'être déjà satisfaisant. Si les quantités de protéines sont à peu près équivalentes, les unités amidon apportées par le silage de queues de betteraves sont près de deux fois et demie plus fortes que celles contenues dans le silage de luzerne.

Si nous additionnons les éléments nutritifs de chacun des deux silages aux éléments nutritifs de la ration fixe de base, nous trouvons :

\begin{tabular}{|c|c|c|c|}
\hline • & $\begin{array}{c}\text { Natière } \\
\text { sèche } \\
g\end{array}$ & $\begin{array}{l}\text { Protéines } \\
\text { digestibles } \\
\text { g }\end{array}$ & $\begin{array}{l}\text { Inités } \\
\text { Amidon } \\
\text { r }\end{array}$ \\
\hline $\begin{array}{l}\text { Ration de base }+ \text { silage luzerne } \ldots \ldots \ldots \ldots . . . \\
\text { Ration de base }+ \text { silage queues de betteraves. }\end{array}$ & $\begin{array}{l}13960 \\
20980\end{array}$ & $\begin{array}{l}\text { I } 887 \\
\text { I } 987\end{array}$ & $\begin{array}{r}7297 \\
10567\end{array}$ \\
\hline
\end{tabular}

Les besoins en protéines digestibles et unités amidon pour des vaches de $500 \mathrm{~kg}$ produisant les quantités de lait indiquées étaient les suivants :

\begin{tabular}{|c|c|c|}
\hline Production de lait (a $35 \%$ ) & Protéines digestibles & $\begin{array}{c}\text { Vunités Amidon } \\
\qquad s\end{array}$ \\
\hline 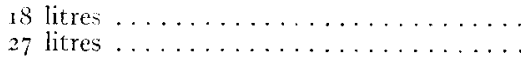 & $\begin{array}{l}\text { I } 200-1290 \\
\text { I } 650-1785\end{array}$ & $\begin{array}{l}7050-7500 \\
9075^{-9} 750\end{array}$ \\
\hline
\end{tabular}

On comprend donc qu'avec le silage de luzerne, vu l'insuffisance d'unités amidon, la production avait tendance à tomber au-dessous de 20 litres et qu'avec le silage de queues de betteraves, elle avait tendance à monter audessus de 20 litres.

Nous examinâmes également les rations données par le Professeur danois HANSEN LARSEN à des vaches produisant 40 à 50 litres et calculâmes les éléments fondamentaux de ces rations, en particulier la matière sèche et le ballast. 
Pour quatre rations, nous trouvâmes:

\begin{tabular}{|c|c|c|}
\hline $\begin{array}{l}\text { Ration Hansex LARSEN pour vaches } \\
\text { produisant } 40-50 \text { litres de lait }\end{array}$ & $\begin{array}{l}\text { Matière sèche } \\
\text { g }\end{array}$ & $\begin{array}{l}\text { Ballast } \\
\mathrm{g}\end{array}$ \\
\hline 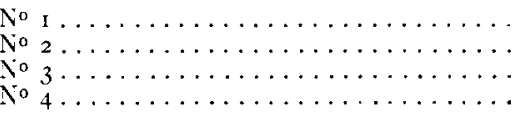 & $\begin{array}{l}23040 \\
10180 \\
23240 \\
22980\end{array}$ & $\begin{array}{l}4970 \\
3700 \\
4840 \\
4730\end{array}$ \\
\hline Moyenne $\ldots \ldots \ldots \ldots$ & 25860 & 4560 \\
\hline
\end{tabular}

Le ballast est donc très voisin de celui indiqué par LEHMANN. Il lui seraiz même inférieur puisqu'il s'agissait de vaches de $650 \mathrm{~kg}$. Par contre la matière sèche est très supérieure, presque $50 \%$ de plus que le chiffre maximum admis.

Il me paraissait donc indiscutable que le ballast expliquait beaucoup mieux que la matière sèche les raisons du rassasiement.

Aux Journées Scientifiques du Lait organisées en I950, à Paris, par le Professeur Terrorne, j'avais eu l'occasion de rencontrer le Professeur CRASEMANn, Directeur de l'Institut pour l'Alimentation du Bétail à l'École Polytechnique de Zürich. Cet éminent Professeur eut l'amabilité de m'envoyer différentes études sur la question de l'alimentation des vaches. En particulier, une brochure du Docteur JUCKER « L'alimentation de la vache laitière en pâturage intensif " et une autre du Professeur CRasemann lui-même : "Le foin de prairie, base de l'alimentation d'hiver des vaches laitières " attirèrent mon attention à nouveau sur la question du ballast. En outre, ces brochures me fournirent différentes références précieuses en particulier celle concernant l'étude fondamentale de Lemmann sur la "Théorie du Ballast".

Je me procurais à la Bibliothèque de 1'Institut Agronomique la Zeitschrift Für Tierernährung Und Futtermittelkunde (Revue pour la nourriture et les aliments du bétail). Dans le tome V de $\mathrm{I} 942$ se trouvait l'admirable et pénétrante étude de LEHMANN "La Théorie du Ballast".

Je lisais bien entendu d'autres articles du même tome. Or, la même année I942, se trouvait une immense étude de F. E. Fissmer sur la question du rassasiement de la vache laitière. Des essais multiples, précis et répétés qu'il avait réalisés, Fissmer déduisait que ni la matière sèche, ni le ballast n'expliquaient le rassasiement de manière satisfaisante; mais que la matière sèche semblait cependant donner une meilleure explication. Il était donc troublant de voir ces essais nombreux contredire la théorie développée cinquante pages plus loin par LeHManN. La théorie de LeHMANN paraissait aussi convaincante que les chiffres de Fissmer, le tout pour aboutir à une contradiction absolue.

Mes méditations m'amenèrent à la conclusion suivante : le ballast explique admirablement les rations de $\mathrm{LARSEN}_{\text {, mes }}$ petites et modestes expériences avec silage de luzerne et queues de betteraves. Donc, cette théorie est vraie dans certains cas.

Je constatais d'autre part dans les essais de Fissmer que la quantité de 
ballast était très souvent inférieure (on peut dire extrêmement inférieure) à celle indiquée par Lehmann. Mais elle ne lui était jamais supérieure (ou de manière insensible). Je faisais la même constatation sur d'autres rations réelles. J'en tirais la deuxième conclusion : La quantité absorbable de $4300 \mathrm{~g}$ de ballast est un maximum qui est atteint dans certains cas, mais peut aussi ne pas être atteint.

Il s'agissait donc de savoir ce qui empêchait l'absorption de cette quantité maxima possible de ballast. Quel était le "goulot " qui freinait ou empêchait cette absorption maxima, possible et réalisée dans de nombreux cas ?

Les études du Professeur Johnstone Wal, $A$ ace à l'Université de Cornell et publiées dans le "Farmers Weeky " semblèrent m'apporter la lumière.

\section{Les essais du Professeur Johnstone-Wallace}

Tous les essais d'alimentation faits avec les vaches pour déterminer les besoins de la vache ou la richesse des aliments sont faits avec des vaches nourries à l'auge. Or, pendant sept à huit mois de l'année, nos vaches pâturent et il n'est pas besoin de démonstrations et calculs compliqués pour comprendre que les conditions d'alimentation sont certainement différentes pour une bête qui pâture et une bête à l'auge.

Le grand mérite des expérimentateurs de l'Université de Cornell fut d'étudier le comportement de la vache qui pâture. Ces essais nous ont apporté des révélations étonnantes et précieuses, sur lesquelles il n'y a pas lieu de s'étendre ici, mais dont j'examinerai seulement certains aspects.

Les recherches firent apparaître d'abord que la quantité maxima d'herbe et de substance nutritive est absorbée quand l'herbe a $10 \mathrm{~cm}$ de hauteur.

On fit brouter pendant trois jours un herbage de $10 \mathrm{~cm}$ de hauteur, et on constata que les vaches mangeaient en moyenne $68 \mathrm{~kg}$ d'herbe par jour.

Pendant les trois jours suivants, sur cet herbage à demi-brouté, on constata que les vaches mangeaient $4 \mathrm{I} \mathrm{kg}$ d'herbe en moyenne par jour.

Puis, pendant les trois jours qui suivirent $\left(7,8\right.$ et $9^{\mathrm{e}}$ jours de pâturage de la parcelle) on constata que sur cet herbage déjà sérieusement "gratté ", les vaches mangeaient $20 \mathrm{~kg}$ d'herbe seulement par jour.

On n'indiquait pas les variations de la composition de cette herbe au fur et à mesure de la progression du broutage, si ce n'est que la teneur en matière sèche de l'herbe était voisine de $2 \mathrm{I} \%$.

Nous prendrons trois caractéristiques de l'herbe dans les tables du Reichsnährstand, nous avons :

\begin{tabular}{|c|c|c|c|c|}
\hline Pour i oos g d'herbe, on a : & $\begin{array}{c}\text { Matière } \\
\text { sèche } \\
g\end{array}$ & $\begin{array}{c}\text { Protéines } \\
\text { digestibles } \\
\mathrm{g}\end{array}$ & $\begin{array}{c}\text { Puités } \\
\text { Amidon } \\
y\end{array}$ & $\begin{array}{c}\text { Ballas }=\mathrm{t} \\
\ddot{4}\end{array}$ \\
\hline I $^{0}$ Rotation I $^{\text {re }}$ qualité. & 180 & $3^{\circ}$ & I I 5 & $3^{6}$ \\
\hline $2^{0}$ Rotation $2^{0}$ qualité & 210 & 23 & 130 & 49 \\
\hline $3^{\circ}$ Pâturage ordinaire. & 220 & I9 & 127 & 58 \\
\hline
\end{tabular}


Nous supposerons que le cas (I) correspond aux 3 premiers jours, le cas (2) aux trois jours suivants, et le cas (3) aux trois derniers jours de pâturage. Nous trouvons alors les quantités absorbées suivantes:

\begin{tabular}{|c|c|c|c|c|}
\hline $\begin{array}{l}\text { Quantités absorbées par les vaches } \\
\text { au cours des } 3 \text { périodes de pâturage }\end{array}$ & $\begin{array}{c}\text { Matière } \\
\text { sèche } \\
g\end{array}$ & $\begin{array}{c}\text { Protéines } \\
\text { digestibles } \\
\mathrm{g}\end{array}$ & $\begin{array}{c}\text { Unités } \\
\text { Amidon } \\
\mathrm{g}\end{array}$ & $\begin{array}{c}\text { Ballast } \\
\mathrm{g}\end{array}$ \\
\hline $\begin{array}{l}\text { Période } 1 \ldots \ldots \ldots \ldots \ldots \ldots \ldots \ldots \\
\text { Période } 2 \ldots \ldots \ldots \ldots \ldots \ldots \\
\text { Période } 3 \ldots \ldots \ldots \ldots \ldots \ldots\end{array}$ & $\begin{array}{r}12200 \\
8620 \\
+400\end{array}$ & $\begin{array}{r}2040 \\
904 \\
380\end{array}$ & $\begin{array}{l}7820 \\
5380 \\
2540\end{array}$ & $\begin{array}{l}2450 \\
2020 \\
1160\end{array}$ \\
\hline
\end{tabular}

Il est évident qu'il y a un cas où la quantité de matière sèche correspond à la quantité théorique ; mais qu'il n'y a aucun cas où la quantité de ballast approche de la quantité maxima possible.

Il y a donc bien eu un goulot qui a empêché l'absorption des quantités maxima possibles de ballast (ou même de matière sèche).

\section{Le rassasiement mécanique}

Les travaux de 1'Université de Cornell nous ont révélé un autre fait inattendu, et, autant que je sache, jusqu'ici inconnu.

La vache passe huit heures environ à pâturer (déplacement et première mastication). Quoi qu'il arrive, elle ne dépasse jamais ce temps. Comme le fait remarquer le Professeur Johnstone-WaLIACE avec humour : "Il semble que le Syndicat des Vaches a imposé à ses membres un strict règlement que toutes les vaches observent scrupuleusement. La journée de pâturage de huit heures n'est dépassée dans aucun cas, même si la nourriture fournie par l'herbage et consommée pendant ce temps est insuffisante ". Autrement dit, la vache passe un temps déterminé à pâturer, puis s'arrête sans s'occuper de savoir si ses organes digestifs sont remplis (rassasiement volumétrique) ou si ses besoins en substances nutritives sont satisfaits (rassasiement physiologique).

La remarque du Professeur JoHnstone-Waldace est fort spirituelle et amusante, mais il est peu probable que les vaches possèdent un chronomètre leur permettant de mesurer exactement leurs huit heures de travail et qu'une sirène d'usine se mette à mugir dans leur tête pour les avertir que le " turbin de la journée est terminé ».

En réfléchissant, j'arrivais à la conclusion suivante :

L,a vache s'arrête parce qu'elle a accompli l'effort maximum dont elle est capable. Elle est fatiguée et ne peut fournir un effort supplémentaire, même si elle a encore faim, et il est certain qu'elle a faim, que 1'on considère le problème du point de vue de la matière sèche ou du point de vue du ballast.

Le "goulot " qui empêche d'absorber les quantités maxima théoriques de ballast est donc l'impossibilité de la vache à dépasser un certain effort ou travail de mastication. 
Il paraît d'ailleurs raisonnable de transformer ce temps constant de broutage en un effort constant, car d'autres mesures de 1'Université de Cornell semblèrent montrer que le nombre de coups de mâchoire à la minute est très régulier et constant.

J'avais d'autant plus tendance à transformer ces temps de broutage (ou de mastication) constants en une dépense d'énergie constante que les travaux de l'École française sous la direction du Professeur LEROY ont déjàattiré notre attention sur un autre point. GASNIER et DELAGE ont en effet constaté que le temps mis par un bovin pour consommer un kilo de matière sèche s'accroît avec la teneur moyenne en fibres (ou cellulose) par kg de matière sèche.

Ils ont trouvé le résultat suivant:

\begin{tabular}{|c|c|c|}
\hline Aliment & $\begin{array}{l}\text { Teneur moyenne } \\
\text { en fibres brutes par kg } \\
\text { de matière sèche } \\
\%\end{array}$ & $\begin{array}{c}\text { Durée de consommation } \\
\text { par kg } \\
\text { de matière sèche } \\
\text { (minutes) }\end{array}$ \\
\hline Paille & 40 & 60 \\
\hline Foin $\ldots \ldots \ldots \ldots \ldots \ldots \ldots \ldots \ldots \ldots$ & 30 & 45 \\
\hline Aliments concentrés (sous forme d'agglomérés). . & 4 & 5 \\
\hline
\end{tabular}

Or, comme le souligne le Professeur LERoy : " Plus le repas est long, plus la perte de métabolisme entraîné par la consommation de l'aliment est considérable ».

Ainsi, à des temps de mastication plus ou moins longs correspondent des dépenses d'énergie plus ou moins grandes. Je croyais donc raisonnable d'admettre qu'à un temps de mastication constant qui d'après les recherches de CoRneli, était limité, pour une vache, par un plafond maximum, correspondait une dépense d'énergie constante, elle-même limitée par un plafond maximum.

Finalement, j'en arrivais à la conclusion :

La théorie du ballast ou rassasiement volumétrique de Lehmann est valable à condition que l'animal ait pu mastiquer la quantité d'aliments correspondant à ce ballast.

Nous appellerons mastication l'ensemble des opérations suivantes principales :

I ${ }^{\circ}$ La préhension des aliments consistant à introduire les aliments dans la bouche. ments.

$2^{\circ}$ La première mastication comportant trituration et broyage des ali-

$3^{\circ}$ La $1^{\text {ere }}$ salivation des aliments.

$4^{\text {o }} \mathrm{La} 1^{\text {ere }}$ déglutition qui fait passer les aliments de la bouche dans le rumen.

$5^{\circ}$ L'humectation par les sucs gastriques.

$6^{\circ}$ La régestion ou remontée des aliments dans la bouche. 
$7^{\circ}$ La $2^{\text {e }}$ mastication.

$8^{\circ} \mathrm{La} 2^{\mathrm{e}}$ salivation.

$9^{\circ}$ La $2^{e}$ déglutition.

Dans le cas d'un animal au pâturage, il faut ajouter à tous ces efforts et dépenses d'énergie :

a) Le cisaillement de la plante.

b) Le déplacement de l'animal pour trouver sa nourriture.

Quoi qu'il en soit, j'estime que le remplissage du système digestif par la quantité maxima possible de ballast ne peut se produire que si l'animal est capable de réaliser le travail de mastication compris dans le sens très général que nous venons d'expliquer.

Je dirai donc qu'il y a "rassasiement mécanique " quand une vache a absorbé la quantité de nourriture pour laquelle elle a dépensé tout l'effort de mastication dont elle est capable.

J'estime que si le rassasiement mécanique n'est pas atteint et que cependant le rassasiement volumétrique est réalisé, la vache s'arrêtera de manger. De même, si le rassasiement mécanique est atteint, même si le rassasiement. volumétrique n'est pas réalisé, elle s'arrêtera de manger.

Autrement dit, ce qui règle la quantité d'aliments absorbés, c'est celui des deux rassasiements qui est le plus vite atteint.

En d'autres termes, on n'a considéré jusqu'ici que le remplissage du système digestif comme critérium du rassasiement. On avait oublié que les aliments passant d'abord par la gueule de l'animal, si la gueule est capable de mastiquer une quantité suffisante d'aliments pour remplir le système digestif, celui-ci sera rempli et son volume règlera la quantité d'aliments absorbés. Si, au contraire, la gueule est fatiguée avant d'avoir mastiqué la quantité d'aliments présentés à l'animal pour remplir le système digestif, c'est la gueule elle-même qui règle la quantité d'aliments absorbés.

Nous considérons la gueule et le système digestif comme deux tubes placés bout à bout. Avec certains aliments, il se trouve que le tube "gueule " a un diamètre plus grand que le "tube système digestif ". Dans ce cas, c'est le tube de plus petit diamètre qui règlela quantité pouvant s'écouler, c'est-à-dire le tube "système digestif ", ce qui signifie que c'est le "rassasiement volumétrique " des organes digestifs qui limitera l'absorption de ce ou ces types d'aliments.

Avec d'autres aliments, le tube "gueule " a un diamètre plus petit que le tube " système digestif ", c'est donc le tube " gueule " qui règle alors le volume d'aliments potuvant s'écouler dans la canalisation; dans ce cas, le travail maximum possible de mastication, autrement dit le "rassasiement mécanique " déterminera la quantité maxima d'aliments pouvant être absorbés.

Telle est ma théorie, qui ne fait que s'ajouter aux multiples théories déjà émises sur la question. Seuls, les résultats pratiques permettront de juger si cette théorie explique mieux les résultats réels que les autres théories. 


\section{Les essais de Fissmer}

Nous reprendrons les études de Fissmer pour essayer de déterminer un critérium permettant de juger de l'action rassasiante d'un aliment ou en même temps des besoins de la vache pour être rassasiée.

FISSMER fournissait à la vache une ou deux rations de base qu'elle devait absorber entièrement, puis on mettait en plus à la disposition de la vache une ration de rassasiement dont elle pourrait manger autant qu'elle voulait. Dans certains essais (comme le montre le tableau récapitulatif de la page 13 ), les rations de base et la ration de rassasiement pouvaient se confondre.

Quoi qu'il en soit, les quantités absorbées de chacune des rations (de base ou de rassasiement) étaient soigneusement pesées. On connaissait donc exactement les quantités maxima d'aliments qu'une vache était capable d'absorber, autrement dit les quantités d'aliments qui rassasiaient la vache. On pourrait dire encore plus exactement : on connaissait les quantités d'aliments après l'absorption desquels la vache se sentait rassasiée.

Nous admettrons comme exacte la théorie de LEHMANN suivant laquelle le ballast de l'aliment est l'élément qui détermine le "rassasiement volumétrique " du système digestif. Le ballast comme nous l'avons déjà dit, est formé par le total des substances organiques non digestibles. Nous admettrons enfin comme exact le chiffre de LEHMANN, de $4,3 \mathrm{~kg}$ de ballast, comme la quantité maxima pouvant être absorbée par le système digestif d'une vache de $500 \mathrm{~kg}$, ce qui correspond à $0,8 \mathrm{I} \mathrm{kg}$ de ballast absorbé par Ioo $\mathrm{kg}$ de poids vif.

Il se trouve que dans aucun des essais de Frssmér, où on n'employait que des aliments volumineux, et relativement fibreux cette quantité de ballast n'a été atteinte. Celle maxima a été de $0,7 \mathrm{I} \mathrm{kg}$ par Ioo $\mathrm{kg}$ de poids vif (essai 4 du tableau récapitulatif de la page I3. Nous avons donc la chance de nous trouver devant une série d'essais, où, d'après notre théorie, si elle est exacte, seul le "rassasiement mécanique " a limité la quantité d'aliments absorbés.

Les cinq séries d'essais ont été faites avec des vaches différentes. Nous supposerons que les vaches de chaque série pouvaient fournir un travail maximum de "mastication" que nous définirons en calories, et que nous appellerons : puissance de mastication, égale à A calories, que faute d'éléments nous admettrons la même pour chaque vache, ce qui est peu probable.

Nous verrons plus loin qu'il est possible de calculer le travail de mastication à partir de certains éléments. Sans nous étendre pour l'instant sur cette formule, nous indiquons qu'elle est la suivante :

$$
\text { Travail mastication }=2,35 \mathrm{~F}\left(\frac{\mathrm{IOO}-\mathrm{W}}{\mathrm{IOO}}\right)-\mathrm{k} \mathrm{B}
$$


Nous prendrons comme il est indiqué plus loin dans nos tableaux :

$$
\begin{aligned}
\mathrm{K} & =0,57 \\
\mathrm{~W} & =43
\end{aligned}
$$

Nous voyons dans le tableau (page I4) de Fissmer, que :

$$
\begin{aligned}
& \mathrm{B} \text { (Ballast) }=4 \mathrm{I} 7 \mathrm{~g}, \text { pour I ooo g de paille. } \\
& \mathrm{F} \text { (Fibres totales) }=366 \mathrm{~g} \text { ! }
\end{aligned}
$$

Nous en déduisons done:

$$
\begin{aligned}
\text { Travail mastication } & =\left(2,35 \times 366 \times \frac{57}{\mathrm{IOO}}\right)-0,57 \times 4 \mathrm{I} 7 \\
& =490-237=253 \text { calories. }
\end{aligned}
$$

Nous prendrons ce chiffre comme base pour étudier les essais de Fissmer (voir le tableau récapitulatif de la page I3).

Soit $x$ le travail de mastication (exprimé en calories) pour un kilo de fourrage de trètle.

Les essais $I$ et 2 nous permettent d'établir l'équation :

$$
\mathbf{I} 6,34 x=7,53 x+(3,57 \times 253)
$$

soit:

D'où :

$$
8,8 \mathrm{I} x=904 .
$$

$$
x=\text { I03 calories }
$$

(tous nos calculs sont faits à la règle, ce qui donne une précision plus que suffisante).

Nous en déduisons que la puissance de mastication moyenne du groupe de vaches utilisé est :

$$
\mathrm{A}=\mathrm{I} 6,34 \times 103=\mathrm{I} 68 \mathrm{o} \text { calories. }
$$

Les essais $3 a, 3^{b}, 3^{c}$ et $3^{d}$, nous permettent d'établir l'équation globale :

$$
\begin{aligned}
& \mathrm{15} 5,0 y+\mathrm{I} 3,7 \times 103=\mathrm{A} . \\
& 30,0 y+\mathrm{I} 3,2 \times 103=\mathrm{A} . \\
& 45,0 y+\mathrm{I} 2,4 \times 103=\mathrm{A} . \\
& 60,0 y+\mathrm{I} 2,2 \times 103=\mathrm{A} . \\
& \hline 150,0 y+5 \mathrm{I}, 5 \times 103=4 \mathrm{~A} .
\end{aligned}
$$

( $y$ désignant le travail de mastication pour $\mathrm{I} \mathrm{kg}$ de betteraves fourragères (du type $\mathrm{r}$ ).

Nous en déduisons :

$$
\begin{aligned}
\mathrm{I} 50 y & =(4 \times \mathrm{I} 680)-(5 \mathrm{I}, 5 \times \mathrm{I03}) \\
& =6720-5300=\mathrm{I} 420
\end{aligned}
$$

d'où :

$$
y=9,5 \text { calories. }
$$

Voyons maintenant quelles sont les variations maxima que nous allons 


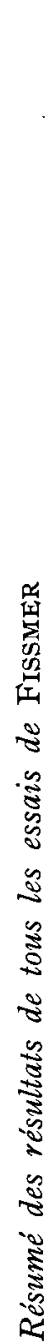

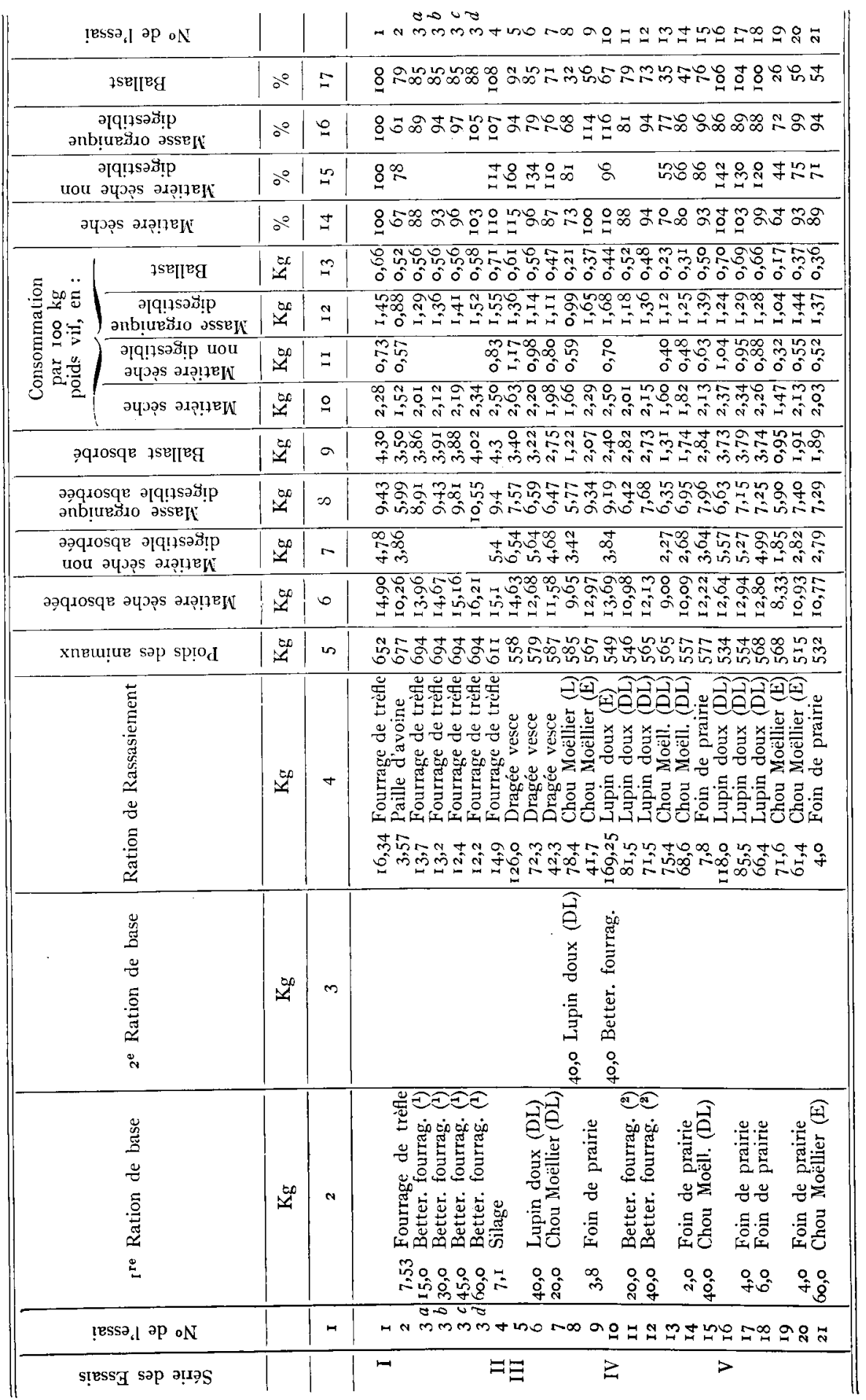

Annalès de Zootechnie. - I952 


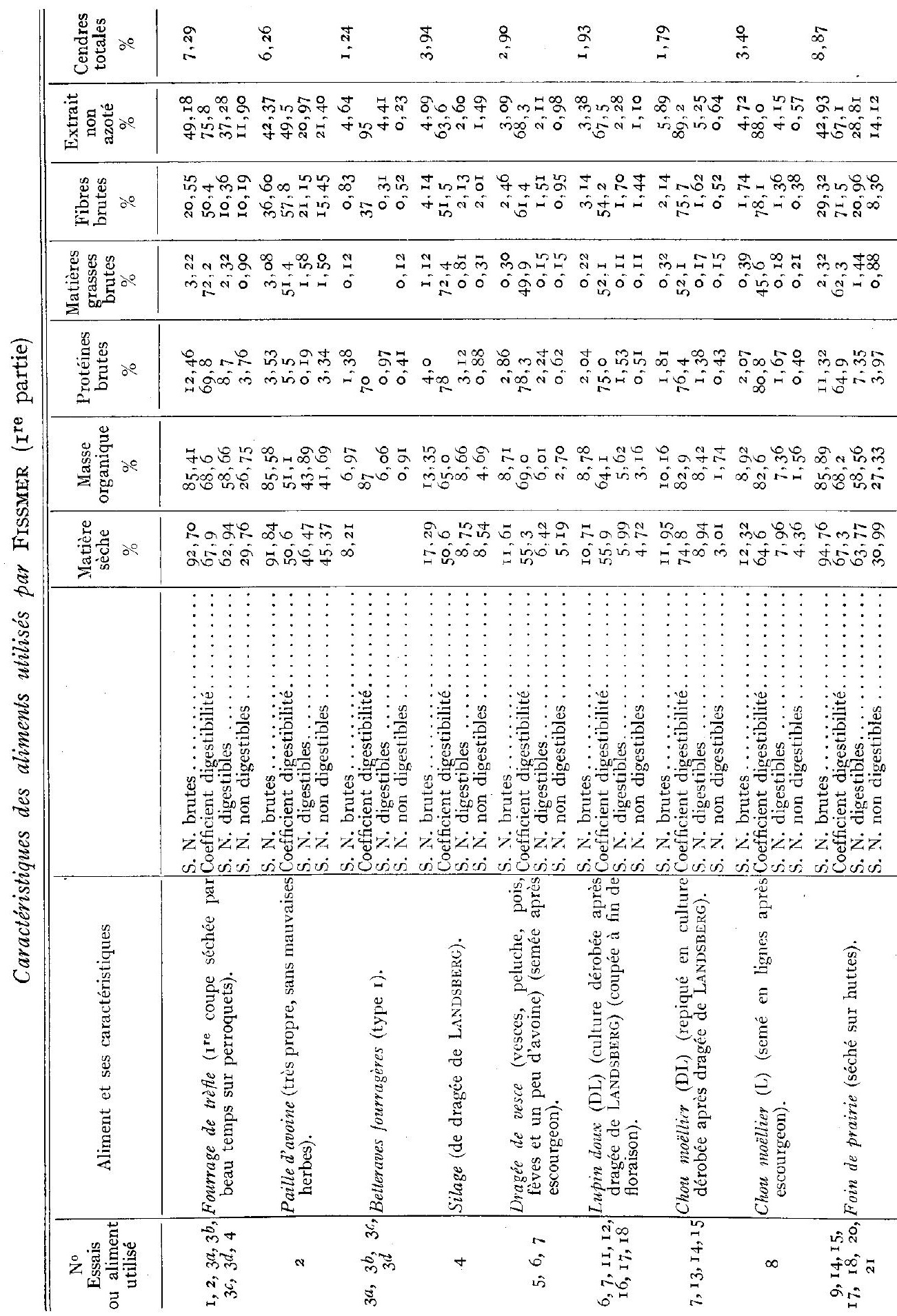


(Sup. I, 1952) QUAND UNE VACHE EST-ELIE RASSASIÉE

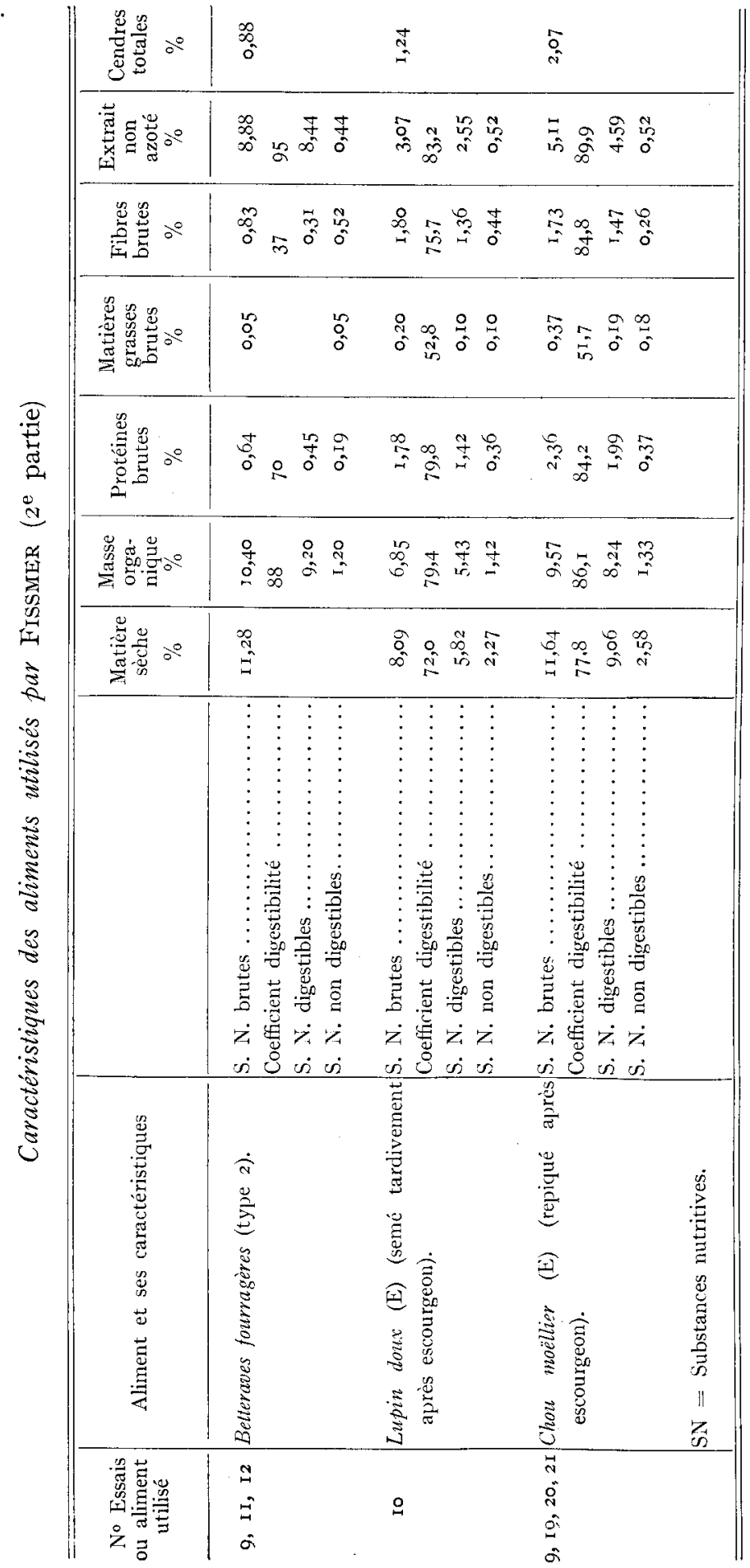


trouver pour le travail de mastication des vaches du groupe utilisé dans la série I des essais.

Nous avons :

$$
\begin{aligned}
& \text { Essai I I6,34 } \times \text { I03 }=\mathrm{I} 68 \mathrm{o} \\
& \text { Essai } 2 \quad(7,53 \times 103)+(3,57 \times 253)=775+904=\mathrm{I} 679 \\
& \text { Essai } 3 a(\mathrm{I} 5 \times 9,5)+\left(\mathrm{I}_{3}, 7 \times \mathrm{IO} 3\right)=\mathrm{I} 42+\mathrm{I} 4 \mathrm{IO}=\mathrm{I} 55^{2} \\
& \text { Essai } 3 b(30 \times 9,5)+(\mathrm{I} 3,2 \times \text { I03 })=284+\mathrm{I} 360=\mathrm{I} 644 \\
& \text { Essai } 3 c(45 \times 9,5)+(\mathrm{I} 2,4 \times \text { I03 })=426+\mathrm{I} 277=\mathrm{I} 703 \\
& \text { Essai } 3^{d}(60 \times 9,5)+(\mathrm{I} 2,2 \times \text { I03 })=568+\mathrm{I} 256=\mathrm{I} 824 \\
& \text { Total }=10082 \\
& \text { Moyenne }=\mathrm{I} 680
\end{aligned}
$$

$\mathrm{Si}$, d'une manière analogue à Fissmer, nous prenons l'essai I comme base roo (ce chiffre correspondant du reste à la moyenne de $\mathrm{I} 680$ calories) nous trouvons :

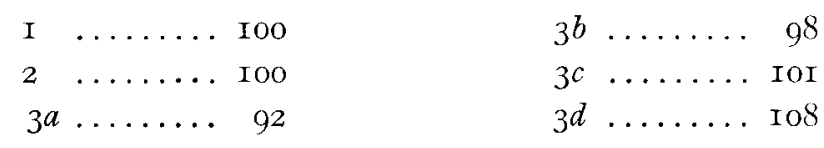

Nous avons donc des écarts maxima de plus ou moins $8 \%$ ce qui est déjà remarquable, mais parait encore plus remarquable quand on voit que l'on trouvait des écarts maxima de $33 \%$ en prenant la matière sèche comme critérium de rassasiement ou de $39 \%$ quand on prenait le ballast comme critérium.

Pour les autres séries d'essais, nous prendrons comme puissance de mastication la même que $\mathrm{A}$ dans la série d'essais I, faute d'éléments.

Nous calculons alors pour les différents aliments, le travail de mastication :

Silage de dRAGÉE de Landsberg (essai 4).

$$
7, \mathrm{I} x+(\mathrm{I} 4,9 \times \mathrm{I0} 3)=\mathrm{I} 680
$$

ou :

$$
7, \mathrm{I} x=\mathrm{I} 680-\mathrm{I} 535=\mathrm{I} 45
$$

et

$$
x=20
$$

DRAGÉE de VESCE

$$
\text { (essai 5) }
$$

$$
\begin{aligned}
\mathrm{I} 26 x & =\mathrm{I} 680 \\
x & =\mathrm{I} 3,3 .
\end{aligned}
$$

LUPIN DOUx (DL)

$$
40 x+(72,3 \times 13,3)=\mathrm{I} 680
$$

d'où :

$$
40 x=\mathbf{r} 680-962=7 \mathbf{I} 8
$$


(Sup. I, I952) QUAND UNE VACHE EST-EILE RASSASIÉE

et

$$
\begin{gathered}
x=\text { I } 8 \\
(\text { essai I6) } \\
\text { I } 8 x=\text { I } 680 \\
x=\text { I } 4,2
\end{gathered}
$$

Nous prenons la moyenne, soit:

$$
x=\frac{\mathrm{I} 8+\mathrm{I} 4,2}{2}=\frac{32,2}{2}=\mathrm{I} 6, \mathrm{I} .
$$

CHOU MÖ̈LLIER (DL) (essai 7)

$$
20 x+(40 \times 16, \mathrm{I})+(42,3 \times 13,3)=\mathrm{I} 680
$$

ou :

$$
20 x=I 680-664-563=433
$$

et

$$
\begin{gathered}
x=2 \mathrm{I}, 6 \\
(\text { essai I3) } \\
75,4 x=\text { I } 680
\end{gathered}
$$

et

$$
x=22,3
$$

Nous prendrons la moyenne soit :

$$
x=\frac{2 \mathrm{I}, 6+22,3}{2}=\frac{43,9}{2}=22 .
$$

BETTERAVES FOURRAGÈres (type 2) (essais II et I2)

Le total de ces deux essais donne:

$$
60 x+(I 53 \times I 6, I)=(2 \times I 680)
$$

ou :

$$
60 x=3360-2470=890
$$

et

FOIN DE PRAIRIE

$$
\begin{aligned}
& x=\mathrm{I} 4,6 \\
& \text { (essai I4) }
\end{aligned}
$$

$$
\begin{aligned}
& \quad 2 x+(68,6 \times 22)=\mathrm{I} 680 \\
& 2 x=\mathrm{I} 680-\mathrm{I} 5 \mathrm{IO}=\mathrm{I} 70 \\
& x=85
\end{aligned}
$$

(essais 17 et 18

I c total de ces deux essais donne:

$$
\begin{gathered}
\text { Io } x+(\text { I } 5 \mathrm{I}, 9 \times \mathrm{I} 6, \mathrm{I})=(2 \times \text { I 680 }) \\
\text { Io } x=3360-2446=9 \mathrm{I} 4 \\
x=9 \mathrm{I}, 4 \quad(\text { essai I } 5) \\
7,8 x+(40 \times 22)=\mathrm{I} 680 \\
7,8 x=\mathrm{I} 680-880=800 \\
x=\mathrm{I} 02 .
\end{gathered}
$$


Nous prendrons la moyenne des trois chiffres, soit:

$$
x=\frac{85+9 \mathrm{I}, 4+\mathrm{IO} 2}{3}=\frac{278,4}{3}=93 .
$$

CHOU MÖ̈LLIER (L)

(essai 8)

$$
\begin{aligned}
78,4 x & =\mathrm{I} 68 \mathrm{o} \\
x & =2 \mathrm{I}, 4
\end{aligned}
$$

LUPIN DOUX (E)

$$
\text { (essai Io) }
$$

$$
\begin{gathered}
\mathrm{I} 69,25 x=\mathrm{I} 68 \mathrm{o} \\
x=9,9
\end{gathered}
$$

Chou Mö̈Llier (E)

$$
\begin{gathered}
\text { (essai I9) } \\
\text { 71,6x = I } 680 \\
x=23,5 \\
\text { (essais 20 et } 2 \mathrm{I} \text { ) }
\end{gathered}
$$

Le total de ces deux essais donne:

$$
\begin{aligned}
& \mathrm{I} 2 \mathrm{I}, 4 x+(8 \times 93)=(2 \times \mathrm{I} 680) \\
& \mathrm{I} 2 \mathrm{I}, 4 x=3360-744=26 \mathrm{I} 6
\end{aligned}
$$

et

$$
x=2 \mathrm{I}, 6
$$

Nous prendrons la moyenne de ces deux chiffres:

$$
x=\frac{23,5+2 \mathrm{I}, 6}{2}=\frac{45, \mathrm{I}}{2}=22,5
$$

Nous trouvons alors le tableau suivant:

Travail de mastication des aliments employés par FISSMER dans ses essais

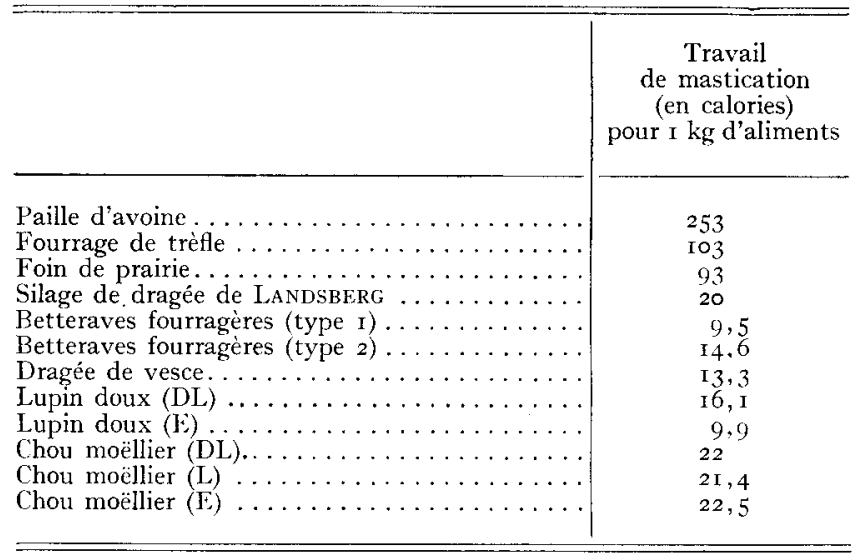

Voyons le travail de mastication total accompli par une vache dans chacun des essais en comparant le résultat obtenu avec le chiffre de l'essai I pris comme base Ioo (I680 calories). 
(Sup. I, I952) QUAND UNE VACHE EST-ELLE RASSASIÉE

Travail total de mastication accompli par une vache dans chacun des essais de Fissner.

\begin{tabular}{|c|c|c|c|}
\hline \multirow[t]{2}{*}{ Essai } & . & \multicolumn{2}{|c|}{$\begin{array}{l}\text { Travail total } \\
\text { de mastication } \\
\text { (en calnries) }\end{array}$} \\
\hline & & calculé & relatif \\
\hline $\mathbf{I}$ & $16,34 \times 103=$ & I.680 & 100 \\
\hline 2 & $(7,53 \times 103)+(3,57 \times 253)=775+904==$ & 1.679 & 100 \\
\hline $3 a$ & $(15 \times 9,5)+\left(13,7 \times 10_{3}\right)=142+1.410=$ & $1.55^{2}$ & 92 \\
\hline $3 b$ & $(30 \times 9,5)+(13,2 \times 103)=284+1.360=$ & I.644 & 98 \\
\hline $3 \%$ & $(45 \times 9,5)+(12,4 \times 103)=426+1.277=$ & 1.7०3 & Ior \\
\hline $3 d$ & $(60 \times 9,5)+(12,2 \times 103)=568+1.256=$ & 1.834 & 108 \\
\hline 4 & $(7,1 \times 20)+(14,9 \times 103)=142+1.535=$ & 1.677 & 100 \\
\hline 5 & $126,0 \times 13,3=$ & 1.680 & 100 \\
\hline 6 & $(40 \times 16,1)+(72,3 \times 13,3)=664+962=$ & I. 626 & 97 \\
\hline $\begin{array}{l}7 \\
8\end{array}$ & $\begin{array}{c}(20 \times 22)+(40 \times 16, \mathrm{I})+(42,3 \times 13,3)=440+664+563 \\
78,4 \times 21,4=\end{array}$ & $\begin{array}{l}1.667 \\
1.680\end{array}$ & $\begin{array}{r}99 \\
100\end{array}$ \\
\hline 9 & $(3,8 \times 93)+(40 \times 14,6)+(41,7 \times 22,5)=352+584+938$ & I.874 & I I 2 \\
\hline Io & $169,25 \times 9,9=$ & 1.680 & 100 \\
\hline I I & $(20 \times 14,6)+(81,5 \times 16,1)=292+1.310=$ & 1.602 & 95 \\
\hline I 2 & $(40 \times 14,6)+(71,5 \times 16, I)=584+$ I.I $50=$ & I.734 & 103 \\
\hline I3 & $75,4 \times 22=$ & I. 657 & 98 \\
\hline I4 & $(2 \times 93)+(68,6 \times 22)=186+1.510=$ & 1.696 & IOI \\
\hline I5 & $(40 \times 22)+(7,8 \times 93)=880+725=$ & 1.605 & 95 \\
\hline 16 & $\mathrm{I} 18 \times 16, \mathrm{I}=$ & 1.900 & I I 3 \\
\hline I 7 & $(4 \times 93)+(85,5 \times 16,1)=372+1.375$ & 1.747 & 104 \\
\hline 18 & $(6 \times 93)+(66,4 \times 16, \mathrm{I})=55^{8}+\mathrm{I} .077$ & I.635 & 97 \\
\hline I9 & $7 \mathrm{~J}, 6 \times 22,5=$ & 1.610 & 96 \\
\hline 20 & $(4 \times 93)+(61,4 \times 22,5)=372+1.390$ & 1.762 & 105 \\
\hline $2 \mathbf{I}$ & $(60 \times 22,5)+(4 \times 93)=1.350+372$ & 1.722 & 103 \\
\hline
\end{tabular}

Nous trouvons donc des écarts maxima de + I3 \%

$$
\text { et }-8 \%
$$

Or, les écarts, avec les méthodes classiques, avaient été de :

$$
\begin{aligned}
& \text { Matière sèche }+\mathrm{I}_{5 \%} \quad \text { Ballast }+6 \% \\
& -36 \% \quad-74 \%
\end{aligned}
$$

Les écarts maxima entre les valeurs maxima et minima étaient done :

$\begin{array}{ll}\text { Travail mastication : } & 2 \mathrm{I} \% \\ \text { Matière sèche : } & 5 \mathrm{I} \% \\ \text { Ballast : } & 80 \%\end{array}$

Il semble donc bien que le rassasiement déterminé par la possibilité maxima de mastication reflète beaucoup mieux la réalité.

Il ne faut pas oublier en outre que nous avons fixé arbitrairement le travail de mastication maximum possible pour les vaches des séries II, III, IV et $\mathrm{V}$ égal à celui déterminé pour la série I grâce au chiffire calculé pour la paille d'avoine.

Il me semble très probable que si nous avions eu la possibilité de déterminer ce chiffre de manière plus précise, nous n'aurions pas dépassé l'écart de $\pm 8 \%$ observé dans la première série d'essais. 


\section{LE COEFFICIENT W DE KELLNER}

Existe-t-il dans nos tables une valeur quelconque qui puisse nous donner une indication sur le travail de mastication, qui, d'après ma théorie, limite l'absorption des aliments tant que la quantité maxima de ballast absorbable n'est pas atteinte?

A la fin de sa carrière, arrivé à l'âge de 80 ans, LEHMANn faisant une analyse pénétrante des théories et chiffres de KELINER, estima que le progrès fondamental que ce savant avait fait réaliser dans la connaissance de nos aliments fourragers était sa découverte de la "Wertigkeit " des aliments, que nous appellerons en français " coefficient d'utilisation " ou plus simplement coefficient " $\dot{W}$ " de KELLNER.

Voyons de plus près en quoi consiste ce coefficient :

Les recherches de KELINER sur l'alimentation des bovins amenèrent une surprise éclatante. Après qu'il eut déterminé la valeur nutritive de chaque substance fondamentale (protéine, matières grasses, amidon) de l'aliment et l'action de chacune de ces substances simples à l'état pur sur l'engraissement des bovins, il décida d'essayer les aliments réels eux-mêmes avec ces mêmes bœufs, et de voir leur efficacité au point de vue de l'engraissement. Il découvrit alors un principe tout à fait nouveau qui peut s'exprimer comme suit:

Des aliments très digestibles, c'est-à-dire avec un coefficient de digestibilité d'environ $90 \%$ des substances organiques avaient une action égale ou presque égale au mélange des substances nutritives pures ; autrement dit, les substances nutritives digestibles de ces aliments sont totalement valorisées. Mais, quand on utilise des aliments moins digestibles, ayant un coefficient de digestibilité de $70 \%$ environ et au-dessous, le poids de graisse réalisé par l'animal ne correspond plus à celui qu'il aurait dû prendre et est inférieur à celui calculé d'après les richesse en substances nutritives digestibles de l'aliment.

Il semblait donc qu'une partie seulement des substances nutritives était utilisée; ce pourcentage des substances nutritives fut appelé par KELLNER "Wertigkeit" ou coefficient d'utilisation et que nous appellerons coefficient W.

Pour prendre quelques exemples, citons le tableau ci-dessous.

\begin{tabular}{|c|c|c|}
\hline Alimenr & $\begin{array}{l}\text { Coefficient de digestibilité } \\
\text { des substances organiques }\end{array}$ & Coefficient \\
\hline 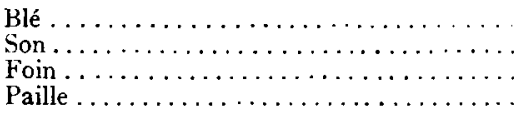 & $\begin{array}{l}86 \\
72 \\
61 \\
42\end{array}$ & $\begin{array}{l}95 \\
77 \\
69 \\
42\end{array}$ \\
\hline
\end{tabular}

Les deux coefficients décroissent donc parallèlement quoique non proportionnellement. 
KELLNER essaya de trouver une relation entre ce coefficient $W$ et la teneur en matières fibreuses de 1'aliment. LEHMANN, au contraire, voulut que ce coefficient $\mathrm{W}$ soit surtout fonction de la teneur en ballast de l'aliment. Nous n'examinerons pas les calculs passionnants et remarquables de ces deux savants et nous contenterons d'examiner certaines des conclusions où LEHMANN semble à peu près d'accord avec KELLNER.

La chute de production réelle par rapport à la production théorique est de (IOO-W) pour cent. Or, KELLNER et LEHMANN ont été d'accord pour admettre que cette chute était due à la dépense d'énergie nécessaire pour le travail de mastication et de digestion. Il en résulte qu'un aliment finement broyé aura un coefficient $W$ plus élevé que le même aliment non broyé, ou en d'autres termes qu'un aliment finement broyé causera une chute de production (IOO-W) plus faible que le même aliment non broyé. KELLNER trouva ainsi que si on avait une chute de production équivalent à $0,5^{8}$ unités amidon pour un fourrage brut, cette chute n'était plus que de 0,29 unités amidon si ce fourrage était finement broyé. Il est évident que ces deux fourrages broyés et non broyés ont la même composition chimique.

LEHMANN, se basant sur les chiffres de KELLNER, essaya de séparer le travail de mastication du travail de digestion, en estimant a priori, que le travail de digestion devait être proportionnel au ballast de l'aliment.

Il arriva aux chiffres suivants qu'il estime du reste très approximatifs vu le peu de précisions données par KELLNER sur le détail de ses expériences :

A) Pour I coo g de matière sèche

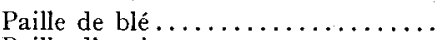

Paille d'avoine $\ldots \ldots \ldots \ldots \ldots \ldots \ldots \ldots \ldots$

Paille d'orge

Moyenne

B) Pour $1000 \mathrm{~g}$ de paille

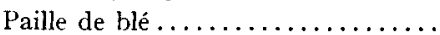

Paille d'avoine $\ldots \ldots \ldots \ldots \ldots \ldots \ldots$

Paille d'orge $\ldots \ldots \ldots \ldots \ldots \ldots \ldots \ldots \ldots$

Moyenne

\begin{tabular}{|c|c|c|c|}
\hline \multicolumn{2}{|c|}{$\begin{array}{l}\text { Travail exprimé } \\
\text { en calories }\end{array}$} & \multicolumn{2}{|c|}{$\begin{array}{l}\text { Travail exprimé } \\
\text { en unités amidon }\end{array}$} \\
\hline Mastication & Digestion & Mastication & Digestion \\
\hline 326 & 345 & I 40 & $14^{3}$ \\
\hline I 24 & 284 & 53 & 122 \\
\hline 115 & 249 & 49 & I I 7 \\
\hline 188 & 25) & $8 \mathrm{I}$ & 129 \\
\hline 277 & 293 & I 19 & 126 \\
\hline 105 & $24 \mathrm{I}$ & 45 & 104 \\
\hline 98 & 212 & 42 & $9 \mathbf{I}$ \\
\hline 160 & 249 & 68 & 107 \\
\hline
\end{tabular}

Nota. - La paille est supposée contenir $86 \%$ de matière sèche.

out

I unité amidon $=2,35$ calories

I calorie $=0,43$ unités amidon.

Il ressort de ce tableau que le travail de digestion est assez constant 
alors que le travail de mastication subit des variations considérables avec les trois espèces de paille.

L,EHMANN prit donc le chiffre moyen de travail de digestion de 249 calories (ou Io7 unités-amidon) par kilo de paille ; et, comme il pensait que ce travail était porportionnel à la teneur en ballast estimée par lui à 435 gr en moyenne par kilo de paille, il en déduisit que le travail de digestion était en moyenne de :

ou

$$
\begin{aligned}
& \frac{229}{440}=0,57 \text { calories par gramme de ballast. } \\
& \frac{107}{440}=0,24 \text { unités amidon par gramme de ballast. }
\end{aligned}
$$

Nous retiendrons ce chiffre de 0,57 calories de travail de digestion pour I gr de ballast. Mais nous ne l'accepterons que dans les conditions où il a été calculé, c'est-à-dire pour des pailles, ce qui signifie pour des aliments avec un $\mathrm{W}$ de 40 environ. LEHMANN a reconnu qu'avec un $W$ croissant il y avait une diminution de la chute de production qui devenait à peu près nulle, tout au moins insignifiante et de l'ordre de grandeur des erreurs expérimentales, pour un W supérieur à 95. Suivant, mais de très loin, un tableau analogue de LEHMANN, nous admettrons les valeurs suivantes pour le coefficient $k$ représentant

\begin{tabular}{|c|c|}
\hline W & $\begin{array}{c}k \text { (calories) } \\
\text { (travail de digestion } \\
\text { par gramme de ballast) }\end{array}$ \\
\hline $95 \dot{a} \quad 100$ & $0,9 \mathrm{I}$ \\
\hline & 0 , I0 \\
\hline $80 \grave{a}$ & 0,20 \\
\hline $70 \mathrm{à}$ & 0,30 \\
\hline 60 a 69 & 0,40 \\
\hline ○ a 59 & 0,57 \\
\hline
\end{tabular}
le travail de digestion en calories par gramme de ballast.

Nous insistons sur le fait que ces chiffres sont purement théoriques et ne peuvent nous permettre que de faire des calculs indicatifs. Seule, l'expérience doit permettre de déterminer ce coefficient $k$.

\section{Le travail de mastication calculé à partir du coefficient $W$}

La chute de production calculée en calories représente d'après KELLNER et LEmManN le travail de nutrition, qui est le total du travail de mastication et du travail de digestion.

La teneur en fibres totales de l'aliment calculée en grammes est multipliée par le facteur $\left(\frac{\text { IOo }-W}{\text { IOo }}\right)$ ce qui donne la chute de production en grammesamidon que nous multiplions par 2,35 pour obtenir la chute en calories ou travail de nutrition : 
Nous avons done:

Chute de production en calories $=\mathrm{F} \times\left(\frac{\mathrm{IOO}-\mathrm{W}}{\mathrm{I} 00}\right) \times 2,35$

$$
\mathrm{F}=\text { teneur totale en fibres. }
$$

D'après LEHMANN, le travail de digestion est:

Travail de digestion en calories $=k \times \mathrm{B}$

$\mathbf{B}=$ Ballast mesuré en grammes.

$k=$ Travail de digestion par gramme de ballast.

Nous en déduisons done:

Travail de mastication $=($ chute de production $)-($ Travail de digestion $)$

(en calories)

$$
=2,35 \mathrm{~F}\left(\frac{\mathrm{IOO}-\mathrm{W}}{\mathrm{IOO}}\right)-k \mathrm{~B}
$$

Nous pouvons inversement calculer $W$. Nous avons en effet:

$$
2,35 \mathrm{~F}\left(\frac{\mathrm{ro0}-\mathrm{W}}{\mathrm{I} 00}\right)=\mathrm{CP} \text { (chute de production en calories) }
$$

d'où

$$
\mathrm{W}=100-\frac{100 \mathrm{CP}}{2,35 \mathrm{~F}}
$$

Eñ résumé :

Connaissant le coefficient $W$, la teneur en ballast et la teneur totale en fibres, nous pouvons calculer le travail de mastication.

\section{Les variations et incertitudes du coefficient $W$}

Le fait grave est l'imprécision régnant sur le facteur W. LEHMAN, dans son analyse pénétrante des travaux et chiffres de KELLNER, a rappelé que KELLNER avait établi ce coefficient $W$ pour pouvoir calculer la valeur alimentaire (unités amidon) des aliments sans avoir à les soumettre à des essais longs et compliqués montrant combien de kilos de viande ces aliments permettent à un bœuf de faire. Le coefficient $\mathrm{W}$ de la plupart des aliments est donc déduit $\mathrm{du} \mathrm{W}$ expérimental d'un aliment analogue, ce qui est déjà une première audace. Mais, la deuxième audace est encore plus grande : KELLLNER admit que la chute de production est rigoureusement proportionnelle à la teneur en fibres totales, ce qui est une hypothèse qui a été loin d'être prouvée par les chiffres. Il suffit du reste de se rappeler que l'état physique d'un aliment (hachage, broyage) influe beaucoup, de l'avis de KELLNER même, sur la chute de production. Vouloir rattacher la chute de production à un élément chimique seul est donc une approximation certainement utile, mais une approximation très approximative. 
On s'aperçoit ensuite que le $\mathrm{W}$ est très variable, suivant les tables, y compris celles de KELINER. Nous avons parlé des essais de KELLNER avec différentes pailles pour lesquelles il a trouvé un certain W. Reportons nous à ses tables et voyons le $W$. Nous avons la comparaison suivante :

\begin{tabular}{|c|c|c|}
\hline & \multicolumn{2}{|c|}{ W } \\
\hline & $\begin{array}{l}\text { Trouvé par les essais } \\
\text { de KeLLNER }\end{array}$ & Tables de Kellner \\
\hline Paille de blé $\left\{\begin{array}{l}\mathrm{a} \ldots \ldots \ldots \ldots \ldots \ldots \ldots \ldots \ldots \ldots \ldots \ldots \ldots \ldots \ldots \ldots \\
\mathrm{b} \ldots \ldots \ldots \ldots \ldots \ldots\end{array}\right.$ & $\begin{array}{l}20 \\
30\end{array}$ & \\
\hline 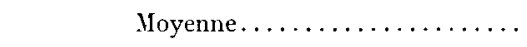 & 25 & $3^{2}$ \\
\hline $\begin{array}{l}\text { Paille d'avoine } \ldots \ldots \ldots \ldots \ldots \ldots \ldots \ldots \ldots \ldots \ldots \ldots \ldots \ldots \ldots \\
\text { Paille d'orge } \ldots \ldots \ldots \ldots \ldots \ldots \ldots \ldots \ldots \ldots\end{array}$ & $\begin{array}{l}60 \\
67\end{array}$ & $\begin{array}{l}43 \\
46\end{array}$ \\
\hline
\end{tabular}

Nous pouvons aussi comparer le $\mathrm{W}$ des tables de KELINER et du Reichsnährstand, nous trouvons :

\begin{tabular}{|c|c|c|}
\hline & & \\
\hline & KeLLNer & Reichsnährstand \\
\hline Paille de blé $\ldots \ldots \ldots \ldots \ldots \ldots \ldots \ldots \ldots$ & 32 & 42 \\
\hline Paille d'avoine $\ldots \ldots \ldots \ldots \ldots \ldots \ldots \ldots \ldots$ & 43 & 42 \\
\hline Paille d'orge $\ldots \ldots \ldots \ldots \ldots \ldots \ldots \ldots \ldots$ & $4^{6}$ & 42 \\
\hline Foin de prairie.. $\left\{\begin{array}{l}\text { mauvais } \ldots \ldots \ldots \ldots \ldots \ldots \\
\text { bon } \ldots \ldots \ldots \ldots \ldots \ldots \ldots \\
\text { excellent } \ldots \ldots \ldots \ldots \ldots\end{array}\right.$ & $\begin{array}{l}49 \\
67 \\
74\end{array}$ & $\begin{array}{l}42 \\
42 \\
42\end{array}$ \\
\hline \begin{tabular}{l|l} 
Pâture verte.... & rotation $\ldots \ldots \ldots \ldots \ldots$ \\
normale $\ldots \ldots \ldots \ldots \ldots \ldots$
\end{tabular} & $\begin{array}{l}9^{2} \\
91\end{array}$ & $\begin{array}{l}72 \\
66\end{array}$ \\
\hline Silage $\ldots \ldots \ldots \ldots, \begin{array}{l}\text { herbe } \ldots \ldots \ldots \ldots \ldots \ldots \\
\text { trèfie violet } \ldots \ldots \ldots \ldots \ldots \\
\text { luzerne } \ldots \ldots \ldots \ldots \ldots \ldots \\
\text { queues de betteraves... } \\
\text { pulpes } \ldots \ldots \ldots \ldots \ldots \ldots\end{array}$ & $\begin{array}{l}75 \\
77 \\
62 \\
92 \\
90\end{array}$ & $\begin{array}{l}69 \\
71 \\
69 \\
69 \\
64\end{array}$ \\
\hline 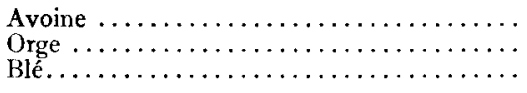 & $\begin{array}{l}95 \\
99 \\
95\end{array}$ & $\begin{array}{l}65 \\
85 \\
98\end{array}$ \\
\hline
\end{tabular}

On voit tout de suite que le Reichsnährstand a simplifié et pris un $W$ de 42 pour tous les fourrages et pailles. Mais, ceci étant noté, on reste inquiet des écarts entre les deux tables.

De tels écarts s'expliquent facilement par la différence des pailles, les tables indiquant seulement une moyenne. Du reste, l'exemple des deux pailles de blé d'hiver essayées par KELLNER montre les écarts formidables de $W$ pour le même type de paille. 
LeHMann a du reste repris les calculs des essais faits par KELLNER avec les pailles et il a trouvé, comme KELLNER du reste :

\begin{tabular}{|c|c|}
\hline ' & $\begin{array}{l}\text { Chute de production } \\
\text { en calories } \\
\text { pour } 1 \text { g de fibres totales }\end{array}$ \\
\hline $\begin{array}{l}\text { Paille de blé } \ldots \ldots \ldots \ldots \ldots \ldots \ldots \ldots \ldots \ldots \\
\text { Paille d'avoine } \ldots \ldots \ldots \ldots \ldots \ldots \ldots \ldots \ldots \ldots \\
\text { Paille d'orge } \ldots \ldots \ldots \ldots \ldots \ldots \ldots\end{array}$ & $\begin{array}{l}1,46 \\
1,05 \\
1,02\end{array}$ \\
\hline
\end{tabular}

Si extraordinaire que cela soit, nous ne pouvons, en appliquant la théorie de KELLNER sur la proportionnalité de la chute de production à la teneur en fibres, retrouver le résultat de ses essais.

\section{Résultats calculés d'après la théorie de Kellner comparés à ceux qu'il a trouvés dans ses essais}

\begin{tabular}{|c|c|c|c|c|c|c|}
\hline & . & & & & & \\
\hline Pour 1 ooo g d'aliments & $\begin{array}{c}\text { Fibres } \mathrm{F} \\
\mathrm{g} \\
\text { (d'après } \\
\text { LEHMANN) }\end{array}$ & $\left\{\begin{array}{c}\text { Ballast B } \\
\mathrm{g}\end{array}\right.$ & $\begin{array}{c}\text { Chute de } \\
\text { production } \\
\text { CP } \\
\text { calories }\end{array}$ & $\begin{array}{l}(100-W) \\
=\frac{100 \mathrm{CP}}{2,35} \mathrm{~F}\end{array}$ & $\begin{array}{l}\text { calculé } \\
\text { d'après } \\
\text { KEILNER }\end{array}$ & $\begin{array}{c}\text { trouvé } \\
\text { par } \\
\text { KeLLNER } \\
\text { dans } \\
\text { ses essais }\end{array}$ \\
\hline $\begin{array}{l}\text { Paille de blé } \ldots \ldots \ldots \ldots \\
\text { Paille d'avoine } \ldots \ldots \ldots \ldots \\
\text { Paille d'orge } \ldots \ldots \ldots \ldots\end{array}$ & $\begin{array}{l}395 \\
335 \\
307\end{array}$ & $\begin{array}{l}4,0 \\
437 \\
403\end{array}$ & $\begin{array}{l}570 \\
376 \\
310\end{array}$ & $\begin{array}{l}6 I \\
44 \\
43\end{array}$ & $\begin{array}{l}39 \\
56 \\
57\end{array}$ & $\begin{array}{l}25 \\
60 \\
67\end{array}$ \\
\hline
\end{tabular}

De sorte que finalement, nous trouvons chez KELLNER même, les W suivants pour les trois espèces de paille.

Paille de blé

Paille d'avoine.

Paille d'orge

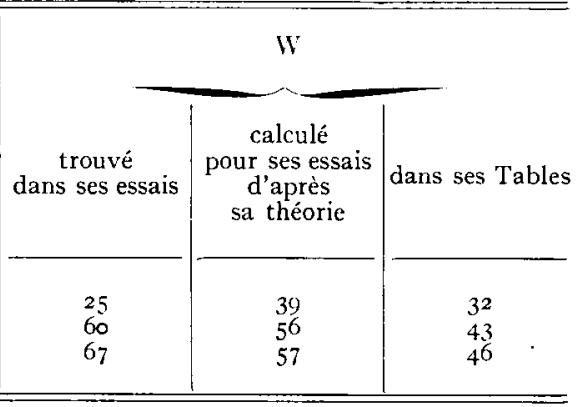

C'est évidemment troublant ; et comme nous ne supposons pas que KELLNER ait fait ses essais ou établi ses tables à la légère, nous devons en conclure que le $W$ varie beaucoup avec la nature de l'aliment : espèce employée, période de coupe, insolation ou pluie pendant la moisson, engrais, etc... 
Il suffit du reste de comparer la teneur totale en fibres indiquée pour ces pailles dans les tables de KFLLNER, ce qui représente une moyenne, et voir la teneur en fibres des pailles utilisées par KELLNER dans ses essais d'alimentation.

\begin{tabular}{|c|c|c|}
\hline & \multicolumn{2}{|c|}{ Teneur totale en fibres pour i ooo g de paille } \\
\hline & $\begin{array}{l}\text { Essais de KELLNER } \\
\mathrm{g}\end{array}$ & $\begin{array}{c}\text { Tables de KELLNER } \\
\mathrm{g}\end{array}$ \\
\hline $\begin{array}{l}\text { Paille de blé } \ldots \ldots \ldots \ldots \ldots \ldots \ldots \ldots \ldots \ldots \ldots \\
\text { Paille d'avoine } \ldots \ldots \ldots \ldots \ldots \ldots \ldots \ldots \ldots \\
\text { Paille d'orge } \ldots \ldots \ldots \ldots \ldots \ldots \ldots\end{array}$ & $\begin{array}{l}395 \\
335 \\
307\end{array}$ & $\begin{array}{l}408 \\
337 \\
395\end{array}$ \\
\hline
\end{tabular}

Il est donc évident que KELLNER a utilisé dans ses essais des pailles d'une qualité exceptionnelle, ayant une teneur en fibres relativement faible, autrement dit des pailles exceptionnellement tendres. Comme il est probable que nos cultivateurs auront très rarement à leur disposition des pailles aussi tendres, nous croyons préférable de prendre les chiffres des tables et utiliserons pour nos calculs les chiffres de fibres totales et de ballast, indiqués dans les tables du Reichsnährstand, qui sont du reste très voisins de ceux de KELINER.

L'éminent professeur Suédois Joël AxELsson qui a fait de nombreuses études sur la valeur nutritive des fourrages verts, secs et ensilés, a estimé qu'on pouvait calculer le coefficient $W$ par l'une des deux formules suivantes:

I) Fourrage vert.

$$
W=99,0-0,428 f-0,0200 f^{2}
$$

2) Fourrage sec et ensilé.

$$
\mathrm{W}=99,0-0,034 f-0,0 \mathrm{I} 9 \mathrm{I} f^{2}
$$

$f=$ le pourcentage de fibres brutes totales calculé par rapport à la matière sèche organique de l'aliment.

L'application de ces formules peut être intéressante dans certains cas; mais, nous avons cru malgré tout préfétable de prendre les vieux chiffres de KELLNER pour le coefficient $W$. Nous connaissons leurs imprécisions et leurs variations, mais nous rappellerons que depuis 50 ans, on les emploie beaucoup sans le dire ou sans le savoir pour calculer les valeurs amidon ou autres unités nutritives.

\section{LES RÉTICENCES DU CULTIVATEUR VIS-A-VIS DES TABLES D'ALIMENTATION}

Nous avons commencé cette étude en parlant des difficultés que rencontrait le cultivateur à utiliser pratiquement les tables d'alimentation. Nous avons estimé qu'une des raisons principales de ces difficultés était le fait qu'on 
avait déterminé les richesses en éléments nutritifs de nos principaux aliments, sans suffisamment étudier les possibilités d'absorption de ces aliments par la vache, autrement dit sans déterminer le ou les critériums déterminant le rassasiement de la vache.

On peut dire que KELLiNER joue vis-à-vis de nos théories de l'alimentation animale un rôle analogue à celui d'Aristote vis-à-vis de la philosophie du Moyen Age. Aussi, dans notre recherche des bases de calcul pour le travail de mastication, nous avons cru bon de nous reporter aux travaux du grand précurseur.

Nous avons alors fait deux constatations principales :

Io Les chiffres des tables concernent un aliment " moyen " et les caractéristiques des aliments réels de même nature peuvent considérablement s'écarter des caractéristiques de cet aliment moyen. Ce n'est pas une constatation nouvelle et originale. Mais, il est bon de souligner que cette variation des aliments réels utilisés par le cultivateur relativement aux caractéristiques moyennes des tables ne facilite pas 1'emploi des tables par le cultivateur.

Il est important de noter à ce sujet une autre observation. Un de nos aliments courants qu'on est en droit de considérer comme ayant les caractéristiques relativement les plus stables est la paille. Or, nous avons vu cidessus les variations énormes du coefficient $W$ pour une même espèce de paille, à savoir la paille de blé (page 24). De telles variations pour un aliment, qu'on peut considérer comme l'un des plus stables, soulignent bien la difficulté que rencontre le cultivateur pour calculer ses rations réelles en utilisant les chiffres moyens des tables.

$2^{\circ}$ La valeur réelle (ou énergie utile vraie) de nos aliments se calcule en unités amidon, à partir de l'énergie totale des éléments nutritifs de ces aliments (déterminée par analyse chimique et essais de digestibilité) d'où on déduit l'énergie dépensée pour la nutrition (mastication + digestion) et qui est proportionnelle à (Ioo-W).

Or, l'analyse chimique et les essais de digestibilité sont des opérations relativement faciles et simples; mais les essais dits de "métabolisme " nécessaires pour déterminer la chute de production (ou énergie de nutrition) sont des essais extrêmement compliqués et difficiles. Même, en Amérique, où les moyens ne manquent pas, ces essais n'ont pu être entrepris que sur une échelle limitée. Il est intéressant de citer à ce sujet, le grand spécialiste américain des questions de nutrition animale, Morrisson, qui dans son monumental ouvrage Feeds and Feeding écrit (pages 5I-52 de l'édition de I947):

\section{AVANTAGES ET LIMITATIONS DE L'ENERGIE UTILE VRAIE}

(net energy value)

“Pendant bien des années les savants ont discuté pour savoir s'il était préférable de comparer les aliments et de calculer les rations sur la base de 
l'énergie totale des substances nutritives (TOTAL DIGESTIBLE NUTRIENTS) ou sur la base de l'énergie utile vraie (NET ENERGY VALUE).

“Il est certain que l'énergie utile vraie donne une idée beaucoup plus exacte de la valeur nutritive des fourrages et autres aliments analogues (ROUGHAGE). C'est cette énergie utile vraie et non l'énergie totale des substances nutritives qui permet de comparer directement ces fourrages avec les grains ou autres aliments concentrés. Par conséquent, de ce point de vue, l'énergie utile vraie est préférable à l'énergie totale des substances nutritives pour estimer la valeur des aliments et calculer les rations de nos animaux.

"Cependant, il y a de grands obstacles scientifiques et pratiques qui limitent l'application de l'énergie utile vraie. Vu le prix extrêmement élevé des essais permettant de déterminer cette énergie utile vraie, il n'a été mesuré cette énergie au moyen d'expériences avec l'appareil respiratoire que pour un nombre très limité d'aliments. Par exemple, quoique la plupart de ces expériences aient été uniquement faites avec des bœufs à l'engraissement, on n'a déterminé jusqu'ici l'énergie utile vraie pour cette classe d'animaux. que pour un peu moins d'une vingtaine d'aliments. On possède encore beaucoup moins de renseignements pour les autres espèces d'animaux.

"Il en résulte donc que l'on est obligé de calculer l'énergie utile vraie de nos aliments à partir des chiffres donnés par l'analyse chimique et les pourcentages de digestibilité. Quand nous procédons ainsi, il est évident que nous faisons des déductions arbitraires, en nous appuyant sur les estimations du savant, de manière à aboutir à des chiffres paraissant raisonnables. Mais quelquefois, les chiffres calculés n'ont pas été corrigés suffisamment par le jugement du savant et diffèrent fortement de ceux observés dans les expériences réelles d'alimentation."

Plus loin (pages 53-54) MORRISON écrit : "Des considérations précédentes, il ressort que l'énergie utile vraie (NET ENERGY VALUE) de nos différents aliments n'a qu'une valeur très limitée comme base pratique de calcul des rations alimentaires de nos animaux. Il faut cependant reconnaitre que la plupart des variations et écarts apparaissant avec cette méthode de calcul se produisent également quand on utilise comme base l'énergie totale des éléments nutritifs (TOTAL DIGESTIBLE NUTRIENTS).

"Il est donc naturel que les opinions des savants diffèrent beaucoup au sujet du système qu'il est préférable d'employer.

"Aux États-Unis, on emploie beaucoup moins qu'en Europe l'énergie utile vraie. La cause en est peut-être qu'on a vu plus clairement les limitation de ce système. Dans notre pays, les valeurs de nos aliments les plus importants ont été déterminées grâce aux nombreux essais d'alimentation réalisés par nos Stations Expérimentales. Les cultivateurs se sont donc appuyés sur les résultats de ces expériences pour déterminer les valeurs relatives des aliments, au lieu de les calculer d'après les énergies utiles vraies.

“Personnellement, j'ai préféré et préfère encore le système del'énergie 
totale des éléments nutritifs (TOTAL DIGESTIBLE NUTRIETS) au système de l'énergie utile vraie (NET ENERGY VALUE). La raison principale de ma préférence vient de ce que l'on possède une quantité très limitée de chiffres concernant l'énergie utile vraie des différents aliments, et que de grandes différences existent pour les chiffres de cette énergie vraie, comme il est apparu dans les pages précédentes. En outre, il semble plus facile pour la plupart des étudiants de comprendre ce qu'est l'énergie totale des éléments nutritifs que le concept de l'énergie utile vraie...

"Malgré tout, nous devons rappeler ce que nous avons déjà dit, à savoir que l'énergie utile vraie, malgré ses limitations, fournit une base beaucoup plus exacte pour comparer les fourrages et pailles aux aliments concentrés. Ceci est également exact quand. on veut comparer un aliment concentré de qualité supérieure ayant une faible teneur en fibres avec un aliment concentré de moindre qualité ayant une teneur assez élevée en fibres. "

Il me paraît indispensable de faire ressortir quelques points importants de cette analyse du grand spécialiste américain.

a) L'énergie utile vraie (ou unités amidon) permet seule de faire une comparaison judicieuse des fourrages avec les aliments concentrés, ou de comparer entre eux, des aliments concentrés ayant des teneurs en fibres différentes. C'était la théorie de KELLNER; elle demeure celle de nos savants modernes les plus éminents.

b) Ces unités-amidon (ou énergie utile vraie) ne peuvent être déterminées qu'au prix d'expériences extrêmement longues et coûteuses. Nous apprenons qu'en I947, c'est-à-dire cinquante ans après les essais de KELLNER, on a soumis tout juste une vingtaine d'aliments à ces essais fondamentaux, et encore avec une seule espèce d'animaux (bœufs à l'engraissement). Il en résulte que l'énergie utile vraie de nos aliments est calculée avec des déductions arbitraires et que le jugement du savant les rectifie quand celui-ci l'estime nécessaire.

c) Ces chiffres d'unités-amidon (ou énergie utile vraie) étant aussi incertains et relativement beaucoup plus variables, Morrisor, comme beaucoup d'autres spécialistes, estime préférable d'utiliser pour ses tables et le calcul des rations l'énergie totale des substances nutritives, quoiqu'il ait lui-même clairement et franchement reconnu que cette énergie ne pouvait fournir une base équitable de comparaison entre de très nombreux aliments.

En réstumé, comme les expériences actuellement réalisées ne permettent pas d'avoir des chiffres suffisamment précis pour l'énergie utile vraie (ou unités amidon) on établit des tables d'alimentation en utilisant 1'énergie totale des substances nutritives, pour laquelle nous possédons des chiffres plus certains mais que nous savons ne représenter la réalité que d'assez loin dans de très nombreux cas.

Nous avons dit au début de cette étude que si le cultivateur montrait tant de réticences à utiliser les tables d'alimentation, c'est qu'il y a quelque chose dans ces tables qui ne "colle" pas. On peut dire que dans ce quelque chose 
se trouve l'imprécision de nos connaissances sur le coefficient $W$ et par suite l'énergie utile vraie, qui est la seule qui intéresse le cultivateur.

\section{Tables de travail de mastication}

A titre d'information, nous avons dressé ci-dessous des tables de travail de mastication, (pages 30 et $3 \mathrm{I}$ ) tout en considérant ces chiffres comme très incertains. Seules, des expériences analogues à celles de FISSMER, permettront d'établir des tables plus certaines, et plus proches des réalités, mais qui, malgré tout, concerneront des moyennes desquelles les aliments réels peuvent fortement s'écarter.

Rappelons que nous calculons le travail de mastication d'après la formivle :

$$
\text { Travail de mastification }=2,35 \mathrm{~F}\left(\frac{\mathrm{I} 00-\mathrm{W}}{\mathrm{IOO}}\right)-k \mathrm{~B}
$$

Le travail de mastication est calculé en calories pour I ooo g d'aliment brut. $\mathrm{F}=$ teneur totale en fibres, mesurée en grammes pour I ooo g d'aliment brut.

$\mathrm{B}=$ teneur en ballast, mesurée en grammes pour I ooog d'aliment brut.

$k=$ travail de digestion en calories pour I $g$ de ballast (variant avec le $\dot{W}$ suivant tableau indiqué plus haut) (page 22).

Travail de mastication déduit des Tables du Reichsnührstand et de Kellner (I er partie)

\begin{tabular}{|c|c|c|c|c|c|c|c|c|}
\hline & $\begin{array}{c}\text { Fibres } \\
\text { F gr }\end{array}$ & $\begin{array}{l}\text { Ballası } \\
\mathrm{B} \mathrm{gr}\end{array}$ & W & $(100-W)$ & $\left(\begin{array}{c}2,35 \mathrm{~F} \\
\left.\begin{array}{c}100-W \\
\text { cal. }\end{array}\right)\end{array}\right.$ & $\mathrm{K}$ & $\begin{array}{l}\text { KB } \\
\text { cal. }\end{array}$ & $\begin{array}{l}\text { Travail } \\
\text { masti- } \\
\text { cation } \\
\text { cal. }\end{array}$ \\
\hline \multicolumn{9}{|l|}{ Verts } \\
\hline Pâtures rotation........... & 39 & $3^{6}$ & 92 & 8 & 7,3 & 0,10 & 3,6 & 3,7 \\
\hline Patures ? normales ........... & $5^{2}$ & 49 & $9 \mathrm{I}$ & 9 & I $\mathrm{I}, 2$ & 0,10 & 4,9 & 6,3 \\
\hline Trèfle jeune à l'automne .... & 42 & $4 I$ & 92 & 8 & 7,9 & 0,10 & $4, \mathrm{I}$ & 3,8 \\
\hline 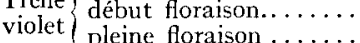 & 43 & 44 & 86 & I 4 & I 4,I & 0,20 & 8,8 & 5,3 \\
\hline rure (pleine floraison ........ & 60 & $5^{6}$ & 83 & I 7 & 24,0 & 0,20 & I I , 2 & I 2,8 \\
\hline 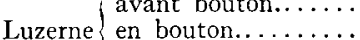 & 36 & 35 & 87 & I3 & I 1,0 & 0,20 & 7,0 & 4,0 \\
\hline en floraison .......... & 80 & $\begin{array}{l}55 \\
90\end{array}$ & 74 & 26 & $\begin{array}{l}27,0 \\
49,0\end{array}$ & 0,30 & $\begin{array}{l}10,5 \\
27,0\end{array}$ & 22,0 \\
\hline Queues et collets de betteraves & 17 & $2 \mathrm{I}$ & 84 & I6 & 6,4 & 0,20 & 4,2 & 2,2 \\
\hline I in floraison...... & 43 & 40 & 88 & I 2 & $12, \mathrm{I}$ & 0,20 & 8,0 & 4,1 \\
\hline Lupm bleu fin floraison ..... & 67 & 63 & $8 \mathrm{I}$ & I9 & 29,9 & 0,20 & 12,6 & 17,3 \\
\hline Dragée de vesce (bouton) .... & 54 & 60 & $8 \mathrm{I}$ & 19 & $24, \mathrm{I}$ & 0,20 & I 2,0 & I 2,1 \\
\hline Silage & & & & & & & & \\
\hline Herbe..$\ldots \ldots \ldots \ldots \ldots \ldots$ & 48 & 43 & 75 & 25 & 28,0 & 0,30 & & I 5.1 \\
\hline Trèfle violet (jeune)......... & 45 & 49 & 77 & 23 & $2,4,4$ & 0,30 & 12,9 & 9,7 \\
\hline Luzerne bouton............ & 39 & 47 & 62 & $3^{8}$ & 34,8 & 0,40 & I 4,7 & 16,0 \\
\hline Luzerne, foraison $\ldots \ldots \ldots \ldots$ & 84 & $9^{2}$ & 57 & 43 & 85,0 & 0,57 & 18,8 & 33 \\
\hline Queues de betteraves......... & 26 & 24 & 92 & 8 & 4,9 & 0,10 & 52,0 & 2,5 \\
\hline Pulpes.................. & 28 & $2 \mathrm{I}$ & 90 & 10 & 6,6 & 0,10 & 2,4 & 4,5 \\
\hline Racines et dérivés & & & & & & & & \\
\hline Betteraves fourragères & Io & 10 & 70 & 30 & $7, \mathrm{t}$ & 0,30 & 3,0 & 4,1 \\
\hline Pulpes fraîches pressées...... & 30 & 30 & 94 & 6 & 4,2 & 0,10 & 3,0 & $\mathrm{I}, 2$ \\
\hline
\end{tabular}


Les valeurs de $\mathrm{F}$ et $\mathrm{B}$ sont prises dans les tables du Reichsnährstand.

Les valeurs de $W$ sont celles des tables de KELLNER.

Il aurait été intéressant de calculer également le travail de mastication pour I ooo g de matière sèche. Malgré le grand intérêt de ce calcul, nous ne l'avons pas fait, de manière à alléger nos tableaux, déjà compliqués et lourds ; d'autant plus qu'il s'agit de tableaux indiquant plutôt les cadres à dresser dans l'avenir grâce à des expériences suivies, et qui ne sont pas destinés à donner des chiffres devant être utilisés dès maintenant par l'éleveur.

Travail de mastication déduit des Tables du Reichsnährstand et Keller (2 ${ }^{\text {eme }}$ partie)

\begin{tabular}{|c|c|c|c|c|c|c|c|c|}
\hline $\begin{array}{c}\text { Pour I ooo gr d'aliment, } \\
\text { on a : }\end{array}$ & $\begin{array}{l}\text { Fibres } \\
F \text { yr }\end{array}$ & $\begin{array}{c}\text { Ballast } \\
\mathrm{B} \text { gr }\end{array}$ & W & $(100-W)$ & $\left(\begin{array}{c}2+35 \mathrm{~F} \\
\frac{100-\mathrm{W}}{\mathrm{1} 00} \\
\text { cal. }\end{array}\right)$ & $\mathrm{K}$ & $\begin{array}{l}\mathrm{KB} \\
\text { cal. }\end{array}$ & $\begin{array}{l}\text { Travail } \\
\text { masti- } \\
\text { cation } \\
\text { ral. }\end{array}$ \\
\hline \multicolumn{9}{|l|}{ Fontrages secs } \\
\hline Foin de, mauvais ...... & 302 & 412 & 49 & $5^{I}$ & $3^{62}$ & 0,57 & 235 & I 27 \\
\hline prairie bon ......... & 285 & $3^{12}$ & 67 & 33 & 221 & 0,40 & 125 & 106 \\
\hline I excellent....... & $23 \mathrm{I}$ & 248 & 74 & 26 & 1.38 & 0,30 & 74 & 64 \\
\hline Trefle, mauvais......... & 297 & $3^{61}$ & 60 & to & 269 & 0,40 & I44 & I 25 \\
\hline violet, bon............. & 282 & 310 & 70 & 30 & 199 & 0,30 & 93 & 106 \\
\hline I excellent......... & 256 & 273 & 74 & 26 & I 57 & 0,30 & 82 & 75 \\
\hline avant boutons & I 94 & $24 \mathrm{I}$ & 71 & 29 & 1.32 & 0,30 & 72 & 60 \\
\hline Luzerne en boutons ... & 268 & 297 & 65 & 35 & 220 & 0,40 & II 9 & 101 \\
\hline I en Heurs........ & 299 & 349 & $5^{2}$ & +8 & 3.38 & 0,57 & 199 & 139 \\
\hline Paillette de blé........ & 308 & 508 & 74 & 26 & 188 & 0,30 & $15^{2}$ & 36 \\
\hline Son de blé............ & 508 & 249 & 77 & 23 & 279 & 0,30 & 75 & 204 \\
\hline Paille d'avoine.......... & 393 & 398 & +3 & 57 & 526 & 0,57 & 227 & 299 \\
\hline Paille rlorge........... & 383 & 407 & 46 & 54 & 486 & 0,57 & 232 & 254 \\
\hline Paille de blé $\ldots \ldots \ldots \ldots$ & 406 & $45^{\mathrm{I}}$ & 32 & 68 & 649 & 0,57 & 257 & 392 \\
\hline $\begin{array}{l}\text { Pulpes séchées .......... } \\
\text { dliments concentrés }\end{array}$ & I 74 & 185 & 78 & 22 & 190 & 0,30 & 56 & I.34 \\
\hline Avoine ............... & 95 & 214 & 95 & 5 & I I, I & $0,0 \mathrm{I}$ & 2 & $9, \mathrm{I}$ \\
\hline Orge $\ldots \ldots \ldots \ldots \ldots \ldots$ & 47 & 104 & 99 & I & $\mathrm{I}, \mathrm{I}$ & $0,0 \mathbf{I}$ & I & $0, \mathrm{I}$ \\
\hline$\ldots \ldots \ldots \ldots \ldots$ & 26 & 99 & 95 & 5 & $3, \mathrm{I}$ & 0,01 & I & $2, \mathrm{I}$ \\
\hline Hails .............. & 22 & 87 & 100 & 0 & 0 & $0,0 \mathbf{I}$ & I & $-I$ \\
\hline Tourteau d'arachide ..... & $5^{6}$ & 125 & 98 & 2 & 2,6 & 0,01 & $\mathrm{I}, 2$ & $I, 4$ \\
\hline Tourteau de Soja....... & $5^{2}$ & 75 & $9^{6}$ & 4 & 4,9 & 0,01 & 0,8 & $4, I$ \\
\hline Tourteau de $\operatorname{Lin} . . . \ldots \ldots$ & 96 & 178 & 97 & 3 & 6,8 & $0,0 \mathbf{I}$ & I, 8 & 5,0 \\
\hline Tourteau de colza...... & IO, & 216 & 95 & 5 & $12, \mathrm{I}$ & 0,01 & $\mathrm{I}, \mathrm{O}$ & I I, I \\
\hline
\end{tabular}

Nous l'avons dit plus haut (et nous ne saurions trop le répéter) que ces chiffres sont purement indicatifs et sont à confirmer par des essais précis. Mais, les essais de FISSMER nous ont donné des chiffres réels pour le travail de mastication. Il est donc intéressant de comparer nos chiffres avec ceux trouvés par les essais de Fissmer.

La concordance est donc acceptable pour les chiffres très faibles (dont nous parlerons plus loin).

Elle est d'autant plus acceptable que nous possédons les analyses exactes des aliments essayés par FISSMER et que les caractéristiques sont loin de concorder exactement avec les chiffres de base que nous avons pris pour le calcul de notre tableau. 
Nous avons donc l'impression que les bases de nos calculs, si elles ne sont pas certaines, ne sont cependant pas trop éloignées de la réalité. Mais, répétonsle une fois de plus, il s'agit de chiffres à vérifier et fixer par l'expérience, et qui, de plus, ne peuvent concerner que des aliments " moyens " desquels

\begin{tabular}{|c|c|c|}
\hline \multirow[b]{2}{*}{. } & \multicolumn{2}{|c|}{$\begin{array}{l}\text { Travail de mastication (en calories) } \\
\text { [pour I ooog d'aliments }\end{array}$} \\
\hline & d'aprés essais de Flisher & Tables ci-clessus \\
\hline P'aille d'avoins $\ldots \ldots \ldots \ldots \ldots \ldots \ldots \ldots$ & 253 & 203 \\
\hline Fourrage de trìle (bon) . . . . . . . . . & 103 & 106 \\
\hline Foin de prairie (bon) ................ & 93 & 106 \\
\hline Silage dragée de LANDSBERG .......... & 20 & \\
\hline Bnage, luzerne (tendre) $\ldots \ldots \ldots \ldots \ldots \ldots$ & & I6 \\
\hline Dragée de vesce $\ldots \ldots \ldots \ldots \ldots \ldots \ldots \ldots$ & 13,3 & I $2, \mathbf{I}$ \\
\hline Lupin $d o u x \ldots \ldots \ldots \ldots$ & $16, \mathbf{I}$ & $17: 3$ \\
\hline 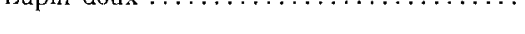 & 9,9 & $4, \mathrm{I}$ \\
\hline Betteraves fourragères............... & $\begin{array}{r}9,5 \\
14,6\end{array}$ & 4,1 \\
\hline
\end{tabular}

les aliments réels peuvent s'écarter très sensiblement. Nous nous consolerons en nous rappelant que la valeur amidon ou la teneur en protéine subissent des variations aussi sensibles par rapport à l'aliment "moyen "indiqué dans les tables.

\section{LES CHIFRES TRES FAIBLES DE MASTICATION}

Nous avons vu dans les essais de FISSMER qu'une vache pouvait manger r69 kg d'un lupin doux très tendre (préalablement coupé et mis dans l'auge), ce qui correspond à un travail de mastication de 9,9 calories. La quantité énorme de lupin absorbé, au premier abord étonnante, correspond bien à ce que des cultivateurs ont pu observer avec des silages de pulpes ou de queues de betteraves hachées, où on peut dépasser largement les roo $\mathrm{kg}$ par vache.

Mais il semble que ce poids de lupin est bien une quantité maxima, autrement dit qu'aucun des aliments peu fibreux et très aqueux puisse être pratiquement considéré comme ayant moins de 10 calories de travail de mastication par kilo. Or, il est évident que parmi ces aliments aqueux, nous en trouvons beaucoup dans ma table, qui ont moins de ro calories, par exemple, le silage de pulpes avec 4,5 et celui de queues de betteraves avec 2,5 calories.

Enfin, nous retrouvons les betteraves fourragères avec $4, \mathrm{I}$ calories. Or, dans les essais de Fissmer, nous avons trouvé pour lè type de betteraves le plus tendre 9,5 calories, ce qui confirme qu'au-dessous de ro calories, nos chiffres doivent être considérés comme n'ayant plus de signification et qu'en principe, le travail de mastication des aliments aqueux, peu riches en fibres, doit être ramené à ro calories minima par kilo. 
Il semble du reste logique que le travail de mastication, compris dans le sens général que nous avons fixé, ne puisse tomber au-dessous d'un certain minimum. Avec un aliment très tendre et parfaitement broyé au préalable, la vache n'aura plus à accomplir le travail de mastication proprement dit, mais il faudra bien qu'elle exerce la préhension, salivage, déglutition, etc... opérations que nous avons inclues dans notre travail de mastication pris au sens général. Il nous apparaît donc raisonnable que ces opérations accessoires de la mastication exigent, quoi qu'il arrive, un minimum de travail. Les chiffres déduits des travaux de Fissmer pour le lupin doux très tendre et les betteraves fourragères peu coriaces indiquent un travail minimum de 9,9 et 9,5 calories, là où notre calcul théorique faisait apparaître $4, \mathrm{I}$ calories. Nous fixerons donc le travail minimum de Io calories par kilo d'aliment aqueux très tendre, chiffre bien entendı qui serait à vérifier par des travaux plus précis et nombreux.

Quant aux aliments concentrés, leur travail de mastication sauf pour l'avoine, et pour le tourteau de colza, est très faible.

Il est du reste à noter que tous ces aliments ont un W supérieur à 90 . Or, Keliner et Lehmann ont toujours été d'accord pour considérer que le travail de mastication de ces aliments était nul ou presque nul.

Fait curieux, il semble qu'avec les moutures, il n'y a presque plus de travail de rumination. L'ouvrage de 1'Institut de Gembloux (page I I6) sur les Aliments du bétail dit à ce sujet :

"Schalk et Amadon ont montré qu'en cas d'alimentation aux grains, une petite fraction seulement est ramenée dans la bouche pour y être de nouveau mastiquée. Comme $50 \%$ seulement ont été écrasés au cours de la première mastication, il en résulte que presque la moitié de la ration de grains traversera telle quelle le tube digestif pour apparaître dans les excréments. Ce sont surtout les aliments fibreux et peu aqueux qui exigent une seconde mastication tandis que pour les aliments aqueux et ceux finement divisés, une seule mastication peut suffire. "

Comme pour les aliments aqueux peu fibreux, nous fixerons un travail de mastication (pris au sens général) de Io calories par kilo d'aliments concentrés. On pourrait objecter que le travail minimum devrait être déterminé au kilo de matière sèche. D'autres, au contraire, pourraient dire que le travail de préhension et déglutition est fonction du poids de l'aliment, qu'il s'agisse d'un réseau sec ou d'un réseau imprégné d'eau. Comme nous ne possédons pour les aliments concentrés aucun travail analogue à celui de FISSMER, il serait difficile d'être certain du chiffre de ro calories. A dire vrai, la chose n'est pas trop grave, car, avec les aliments concentrés, le travail de mastication joue un rôle tout à fait secondaire en ce qui concerne le rassasiement, et c'est le ballast qui limite la quantité d'aliments absorbés. Nous allons donc maintenant examiner ce problème. 


\section{Rapport entre le ballast et le travail de mastication}

Il est intéressant de savoir si un aliment a son absorption limitée par le travail de mastication ou par le ballast.

Il est peu intéressant de savoir les quantités maxima qu'une vache pourrait absorber si elle mangeait uniquement cet aliment, ce qui est un cas exceptionnel et peu recommandable. Mais, il est important de voir si l'absorption d'un aliment est limitée plutôt par la mastication ou plutôt par le ballast, de manière à obtenir un équilibre entre les deux goulots tout en apportant le maximum de protéines et d'unités amidon dans le rapport voulu.

Nous avons donc dressé les tables des pages suivantes (p. 35, 36, 37 et 38) d'après les travaux de mastication trouvés dans les tables précédentes (mais en ne les faisant jamais tomber au-dessous de 10 calories) et d'après les tables du Reichsnährstand pour les éléments nutritifs et le ballast.

Voyons le travail de mastication total accompli par une vache dans chacun des essais en comparant le résultat obtenu avec le chiffre de l'essai I pris comme base roo (I 680 calories).

Nous pouvons en déduire le classement en deux catégories.

\section{A. - Aliments dont l'absorption est limitée par le ballast.}

(Ce sont ceux avec lesquels, pour I680 calories dépensés à les mastiquer, il a été absorbé plus de $4300 \mathrm{~g}$ de ballast).

Aliments concentrés.

Silages (sauf queues de betteraves et pulpes).

Pulpes fraîches.

Foin de prairie mauvais et bon.

Fourrage de trèfle violet.

Fourrage de luzerne, avant boutons et en boutons.

Paillette de blé.

Tous les verts.

\section{B. - Aliments dont l'absorption est limitée par le travail de mastication.}

(Ce sont ceux avec lesquels pour I680 calories dépensés à les mastiquer

il a été absorbé moins de $4300 \mathrm{~g}$ de ballast).

Queues de betteraves fraîches.

Queues de betteraves ensilées.

Silage de pulpes.

Betteraves fourragères.

Fourrage de luzerne en fleurs.

Foin de prairie, excellent. 
(Sup. I, I952) QUAND UNE VACHE EST-ELLE RASSASIÉE

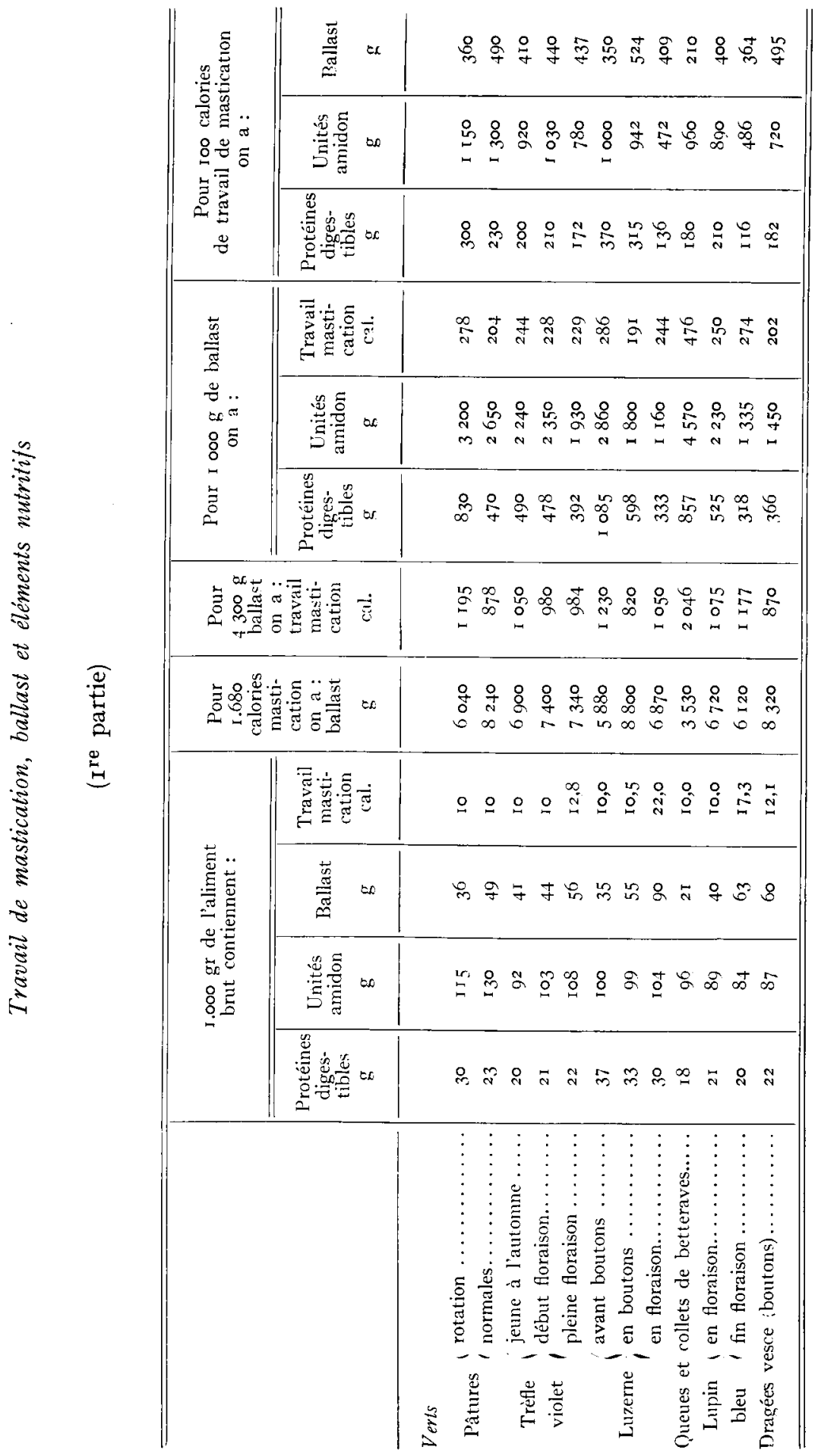




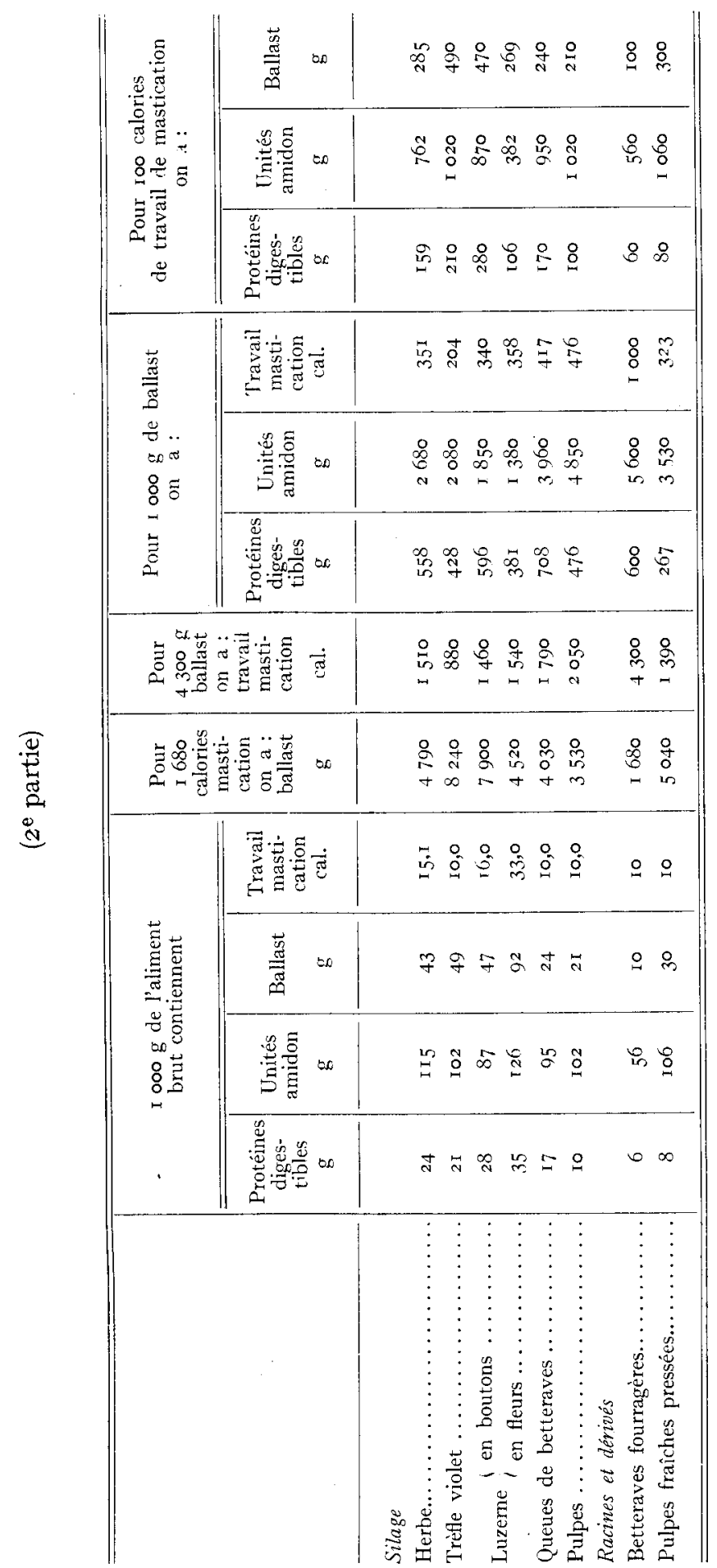


(Sup. I, I952) QUAND UNE VACHE EST-ELLEE RASSASIÉE

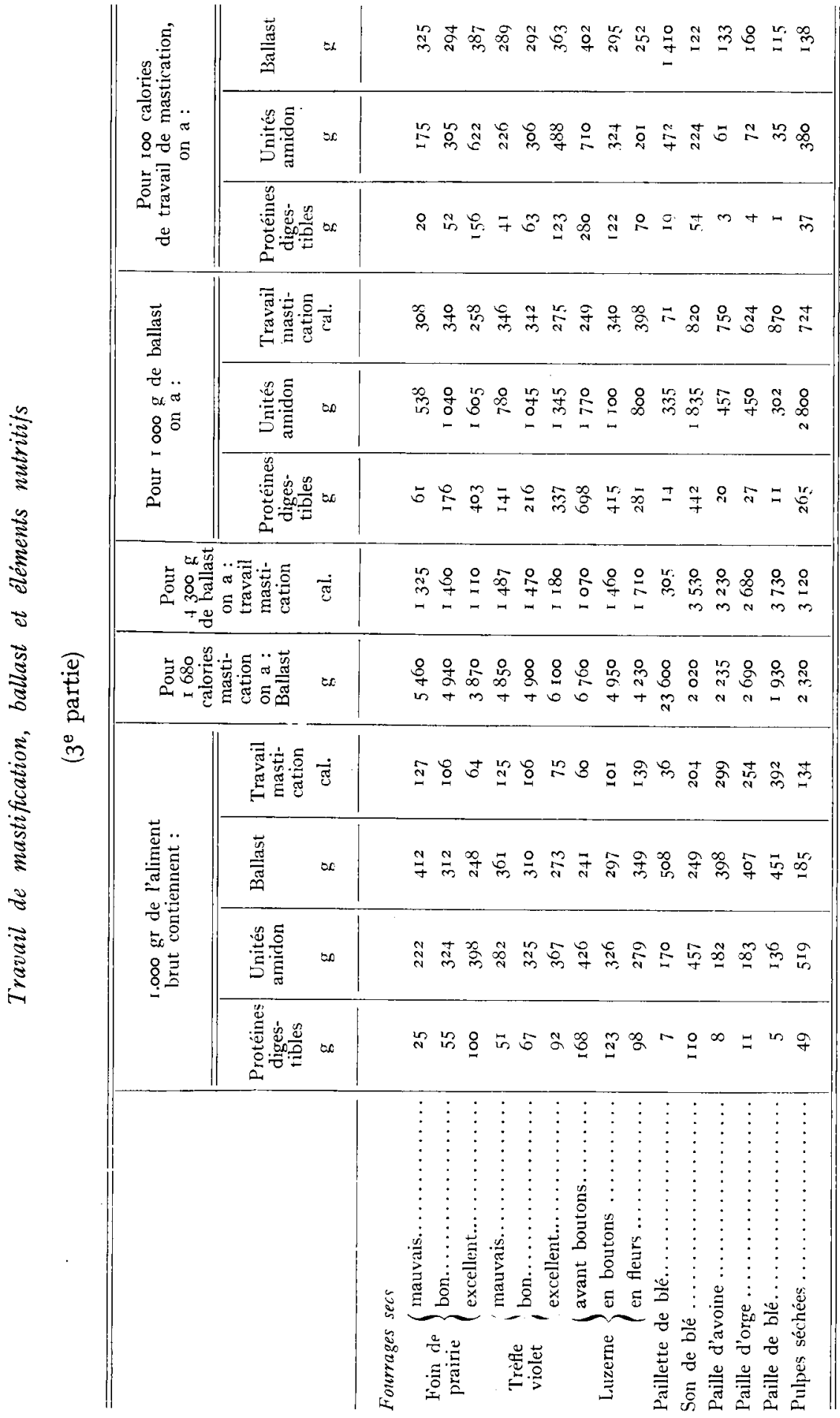


A. VOISIN

(Sup. I, I952)

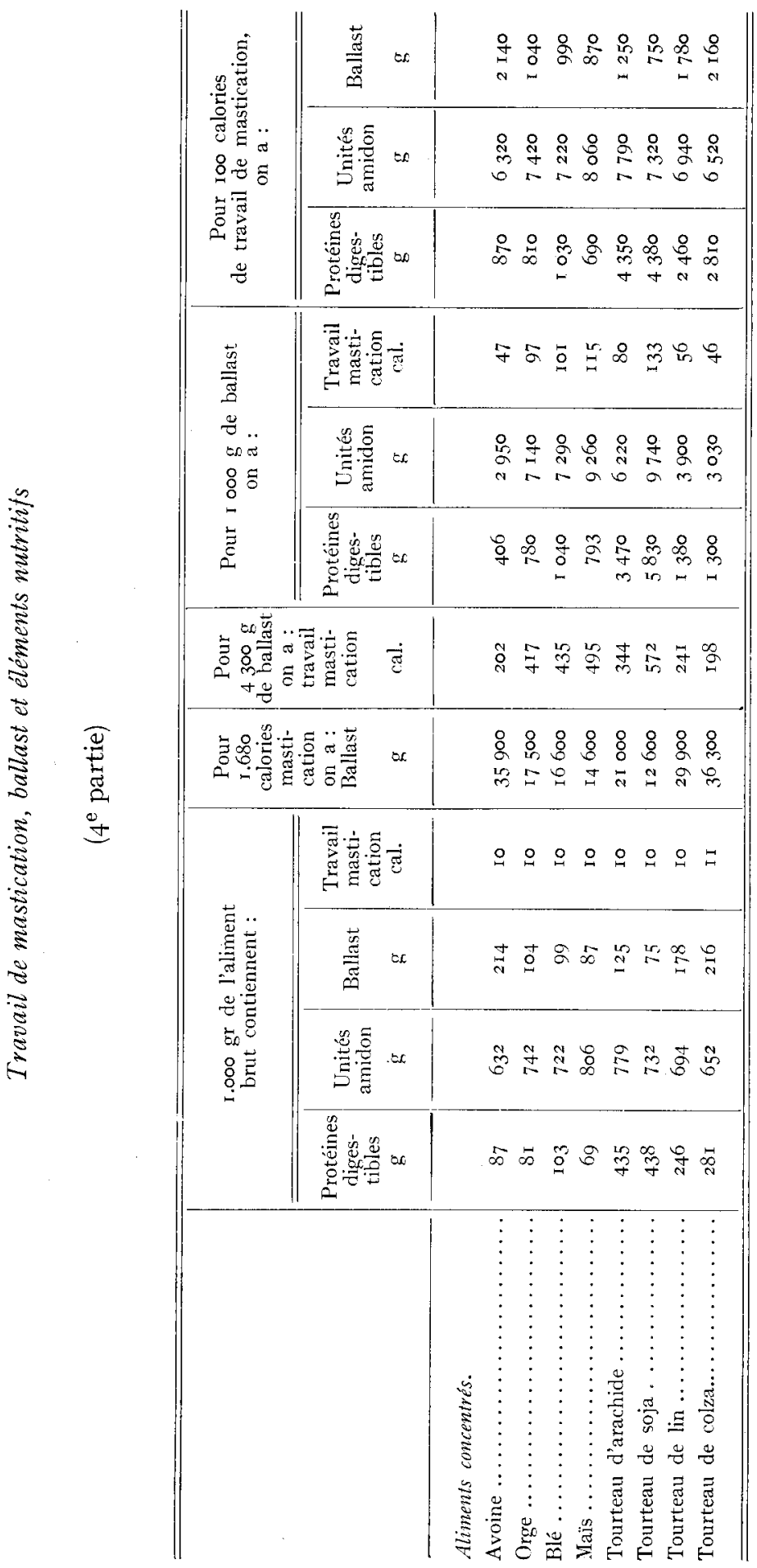


Son de blé.

Toutes les pailles.

Pulpes séchées.

On voit immédiatement une contradiction avec les essais de FIssmer où l'absorption de fourrage de trèfle violet, de lupin doux vert (đe deux sortes) et de dragée de vesce verte était limitée par la mastication.

Mais quand on compare les chiffres de ballast des tables du Riechsnährstand avec ceux des aliments employés par FISSMER, on trouve la comparaison suivante :

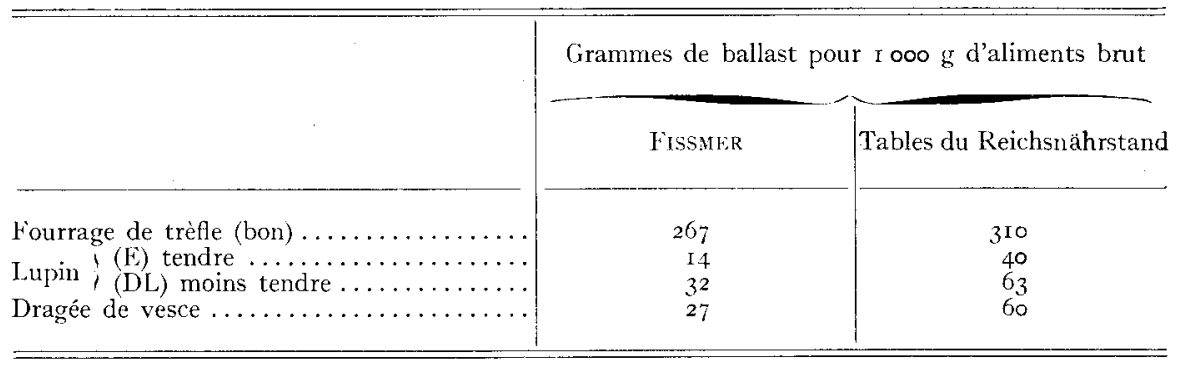

On comprend immédiatement que les aliments " moyens " des tables courantes beaucoup plus riches en ballast, soient limités dans leur absorption par le ballast. Au contraire, les aliments employés par FISSMER, faits en culture dérobée, étaient d'une teneur exceptionnellement faible en ballast; il était donc normal que leur absorption ait été limitée par la mastication.

Notons une fois de plus combien les aliments réels peuvent varier par rapport aux aliments moyens des tables, et que toutes les tables, si bien établies qu'elles soient, peuvent être seulement un guide indicatif pour le cultivateur dans ses méthodes d'alimentation.

\section{LES ENSEIGNEMENTS PRATIQUES}

Il est d'abord nécessaire de rappeler des vérités évidentes trop souvent oubliées.

La vache n'est pas une machine dont on peut régler la consommation de carburant (aliment) à volonté ou faire tourner à un nombre de tours (litres de lait) déterminé comme on pourrait le prévoir d'après les calculs.

Nous ne mesureron jamais la puissance de mastication de la vache comme nous sommes capables de mesurer la puissance d'un moteur. Nous ne déterminerons jamais la capacité d'absorption de ballast d'une vache comme nous jaugeons le volume d'un réservoir.

Nous ne devons jamais oublier que la vache est un être vivant qui a besoin d'être suivie et soignée par un autre être vivant, qu'il s'appelle vacher ou éleveur. 
Nos tables d'alimentation, nos rations-types ne devront jamais être considérées que comme des guides mais jamais comme des équations infaillibles ou des règles absolues.

La combinaison des tables d'alimentation et de l'œil de l'éleveur peuvent donner des réstultats magnifiques. Les tables d'alimentation seules peuvent amener à des désillusions et à des résultats fort médiocres.

Prenons un exemple simple et frappant qui résulte de notre étude : KELLNER et LEHMANN ont montré que des pailles ou fourrages finement broyés permettaient de diminuer considérablement la "perte d'utilisation" ou plus exactement d'augmenter le coefficient $W$. Une conclusion évidente en découle : broyons finement nos aliments fibreux, qui sont des aliments bon marché, produits à la ferme et dont nous disposons généralement en quantité abondante. Achetons des broyeurs à marteaux, des moulins à plateau, etc. Nous faisons une très belle farine. Mais, pour peu qu'il s'agisse d'un fourrage très sec et que nous ayons broyé très finement, la farine risque de ressembler à de la poussière. Les vaches s'englueront la langue, souffleront sur la nourriture en envoyant de la poussière dans toutes les directions et finalement " gabilleront " cette poussière astringente et engluante sans en manger beaucoup.

Nos tables ne pouvaient nous indiquer ce phénomène.

Nous ne verrons jamais dans nos tables si un aliment est mangé avec avidité ou non par la vache. Or, personne ne niera que c'est là un facteur important.

Ne cherchons donc pas des enseignements rigoureux dans nos tables, mais des indications qui sont du reste fort précieuses.

Les deux théories combinées $d u$ "rassasiement mécanique " (travail de mastication maximum possible) et du " rassasiement volumétrique " (quantité maxima possible de ballast) doivent certainement permettre de rendre plus pratiques et réelles nos tables et nos rations-types.

Nous pouvons aussi tirer des considérations ci-dessus des enseignements qui sont loin du reste pour la plupart d'être nouveaux.

Il est indispensable de récolter des fourrages secs ou ensilés avec le minimum de fibres et de ballast. Ce sont seulement les plantes jeunes qui peuvent nous permettre d'obtenir de tels fourrages.

Dire qu'une jeune luzerne contient $3,7 \%$ de protéines digestibles alors qu'une luzerne en floraison contient 3,0\% de protéines digestibles est certainement une indication précieuse. On en déduit qu'on donnera une nourriture $20 \%$ plus riche en protéines digestibles si on apporte à l'auge de la jeune luzerne verte au lieu de la luzerne verte plus âgée. En fait, on est très loin d'exprimer la réalité avec ce chiffre de $20 \%$.

Dans le cas de la luzerne en fleurs, l'absorption est limitée par le ballast (rassasiement volumétrique). Or, avec une jeune luzerne, pour I ooo g de ballast, la vache aura absorbé $\mathrm{I} 085 \mathrm{~g}$ de protéines digestibles, alors qu'avec 
une luzerne plus âgée pour I ooo g de ballast, la vache aura absorbé setulement $333 \mathrm{~g}$ protéines digestibles. Nons sommes loin des $20 \%$; il s'agit d'un rapport de un à trois.

On comprend alors l'influence formidable que peut avoir sur la production laitière une faible variation des caractéristiques de la plante, par suite d'un degré plus ou moins avancé du stade de croissance de cette plante.

Prenons ensuite deux fourrages secs dont l'absorption est limitée par le travail de mastication (rassasiement mécanique). Prenons un foin de prairie excellent dont la teneur en protéines digestibles est de 9,8\%. Manquant de ce forn, nous décidons de le remplacer par du fourrage de luzerne, coupé à la floraison (comme c'est le cas la plupart du temps). Comme la teneur en protéines digestibles de ce fourrage est de I0,0 \% (soit pratiquement identique à la teneur du foin de prairie) nous ne risquons pas de faire absorber moins de protéines à la vache. Mais le travail de mastication est de 64 calories par kilo de foin de prairie alors qu'il est de I39 calories pour le fourrage de luzerne (en fleurs). Il en résulte que pour roo calories de travail de mastication, nous faisons absorber à la vache $156 \mathrm{~g}$ de protéines digestibles dans le cas du foin de prairie alors qu'avec notre luzerne, nous ne lui en feronsabsorber que 70 calories. Il s'agit d'une différence du simple au double.

L'enseignement fondamental est donc: utiliser des aliments avec le moins de ballast possible ou exigeant le plus petit travail de mastication possible.

Une solution simple et connue est de récolter des plantes très jeunes. Plusieurs questions se posent de suite :

$I^{0}$ Le fanage naturel des très jeunes plantes est quasi impossible dans nos régions. Il faut donc utiliser des méthodes de séchage artificiel fort onéreuses. Ces dépenses sont-elles compensées par des avantages ?

$2^{\circ}$ L'ensilage des très jeunes légumineuses est difficile. Il faut mettre aut point les techniques: flétrissage préalable, mélange avec des nourritures riches en hydrocarbones et sucres, etc... permettant de réunir facilement et économiquement cet ensilage.

$3^{\circ}$ Couper une plante fourragère très jeune signifie multiplier le nombre de coupes, ce qui souvent amène des diminutions de rendement à 1'hectare. Cette diminution de rendement sera-t-elle compensée par les avantages ?

$4^{\circ}$ Les très jeunes plantes vertes causent souvent des accidents intestinaux. Il faut étudier le moyen de les empêcher en apportant des aliments (grain, etc...) qui ralentissent la vitesse du "flux " alimentaire.

Pour nous autres cultivateurs, il nous paraîtrait utile d'être éclairés sur ces questions, et nous examinerons plus loin les recherches que nous croyons intéressant d'entreprendre pour nous aider dans l'établissement de nos rations.

Auparavant, nous allons étudier rapidement quelques rations en nous appuyant sur notre théorie des deux rassasiements "volumétrique " et " mécanique " combinés et interdépendants. 


\section{Etude de quelques rations}

Examinons une ration pour vache de $I_{5}$ litres de lait (à $35 \%$ de matière grasse) dont les besoins quotidiens sont:

$$
\begin{array}{ll}
\text { Protéines digestibles : } & \text { I } 050 \text { à I I } 25 \mathrm{~g} \\
\text { Unités Amidon : } & 6375 \text { à } 6750 \mathrm{~g}
\end{array}
$$

Nous indiquons ci-dessous une ration classique de nos manuels d'alimen-

\begin{tabular}{|c|c|c|c|c|c|}
\hline & $\begin{array}{c}\text { Matiòre } \\
\text { sèche } \\
8\end{array}$ & $\begin{array}{c}\text { Protéines } \\
\text { digestibles } \\
\mathrm{g}\end{array}$ & $\begin{array}{c}\text { Unités } \\
\text { Amidon } \\
g\end{array}$ & $\begin{array}{c}\text { Ballast } \\
\mathrm{g}\end{array}$ & $\begin{array}{c}\text { 'Travail } \\
\text { mastication } \\
\text { cal. }\end{array}$ \\
\hline $20 \mathrm{kgs}$ silage pulpes......... & 2800 & 200 & 2040 & 420 & 200 \\
\hline Io kgs betteraves fourragères.......... & I 000 & 60 & 560 & 100 & 100 \\
\hline $2 \mathrm{kgs}$ fourrage de luzerne (en boutons)... & I 720 & $2+5$ & 652 & 594 & 202 \\
\hline 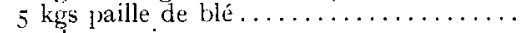 & 4300 & 25 & 680 & 2255 & 1750 \\
\hline 2,5 kgs avoine $\ldots \ldots \ldots \ldots \ldots \ldots \ldots \ldots \ldots$ & 2150 & 217 & I 580 & 545 & 25 \\
\hline \multirow[t]{2}{*}{$\mathrm{I}, 5 \mathrm{~kg}$ tourteau de lin $\ldots \ldots \ldots \ldots \ldots \ldots$} & I 335 & $3^{60}$ & $\mathrm{I} \mathrm{O}_{4} \mathrm{I}$ & 297 & I 5 \\
\hline & I3 305 & 1117 & 6553 & 42 I I & 2292 \\
\hline
\end{tabular}
tation :

Les quantités de protéines digestibles et unités amidon correspondent bien à celles exigées pour I 5 litres. La matière sèche se situe entre $I_{2}$ et $I_{5} \mathrm{~kg}$, et le ballast est inférieur à $4300 \mathrm{~g}$. Tout semblerait donc devoir bien aller. Mais, à notre point de vue, le travail de mastication est beaucoup trop élevé et la vache ne pourra pas absorber cette ration. Il faut donc réduire ce travail en diminuant la quantité de paille ; nous augmenterons légèrement le fourrage de luzerne. Comme celui-ci est plus riche en protéines, nous pourrons en pro-

\begin{tabular}{|c|c|c|c|c|c|}
\hline & $\begin{array}{c}\text { Matière } \\
\text { sèche } \\
\text { g }\end{array}$ & $\begin{array}{c}\text { Protéines } \\
\text { digestibles } \\
g\end{array}$ & $\begin{array}{l}\text { Uuités } \\
\text { Amidon } \\
\text { g }\end{array}$ & $\begin{array}{c}\text { Ballast } \\
\mathrm{g}\end{array}$ & $\begin{array}{c}\text { Travail } \\
\text { mastication } \\
\text { cal. }\end{array}$ \\
\hline $20 \mathrm{kgs}$ silage pulpes........... & 2800 & 200 & 2040 & 420 & 200 \\
\hline Io kgs betteraves fourragères............ & 1000 & 60 & 560 & 100 & 100 \\
\hline 4 kgs fourrage de luzerne (en boutons)... & 3440 & 492 & I 304 & 1 I 88 & 404 \\
\hline 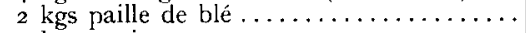 & 1720 & 10 & 272 & 902 & 784 \\
\hline \multirow[t]{2}{*}{ 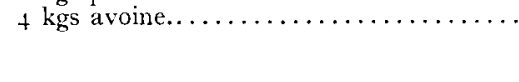 } & 3440 & $34^{8}$ & 2528 & $85^{6}$ & 40 \\
\hline & I 2400 & I IIO & 6704 & 3466 & I 528 \\
\hline
\end{tabular}
fiter pour supprimer le tourteau de lin. Nous arrivons alors à la ration suivante :

Comme précédemment, matière sèche, protéines digestibles, unités amidon et ballast conviennent. Mais, cette fois, le travail de mastication ne dépasse pas les possibilités de la vache et celle-ci pourra avaler la ration, ce qui est la condition première pour qu'une ration convienne. 


\section{La vache au pâturage}

Nos Tables concernent l'alimentation de la vache à l'auge, c'est-à-dire une période qui dure environ cinq mois dans nos régions. Autrement dit, nos Tables ne nous servent théoriquement pas pendant la plus grande partie de l'année.

Il est donc encore bien plus important de déterminer les méthodes rationnelles de pâturage. Dans le cas des grandes vaches laitières, que la sélection rend de plus en plus courantes, les méthodes de pâturage doivent être perfectionnées et exigent pour utiliser les possibilités maxima du pâturage intensif et de la grande laitière, une alimentation complémentaire.

Le sujet est fort vaste. Contentons-nous de dire que le " rassasiement mécanique " y joue un gran đ rôle, étant entendu que le travail de mastication inclut dans ce cas le cisaillement de la plante et le déplacement de la vache. Ce travail sera donc infiniment plus grand qu'à l'auge, pour la même plante verte préalablement coupée.

La question est si vaste que nous nous arrêterons là.

\section{LES RECHERCHES A ENTREPRENDRE}

Si notre hypothèse du rassasiement mécanique est exacte, il semble nécessaire que les Centres de recherches entreprennent des essais pour nous éclairer sur certains points concernant ce rassasiement :

\section{$1^{o}$ Travaux concernant la mastication elle-même de la vache.}

a) Vérifier en employant la méthode indirecte du rassasiement, comme l'avait fait FISSMER, que le travail de mastication est une constante pour une vache donnée dans des conditions analogues.

b) Voir les facteurs extérieurs qui modifient cette puissance de mastication : température extérieure, humidité de 1'atmosphère, intensité du soleil, etc.

c) Y a-t-il une relation entre la capacité de mastication de la vache et certains caractères physiques de l'animal : poids, dimensions de la mâchoire, âge, race, etc...

d) Est-il possible d'augmenter la capacité de mastication de la vache par certaines méthodes correspondant à une espèce de "culture physique de la mastication ": aliments fournis dans le jeune âge, accoutumance à la mastication d'aliments durs et fibreux ?

e) Vérifier la théorie de LEHMANN d'après laquelle le travail de digestion est fonction du ballast. Déterminer cette fonction.

f) Voir s'il existe une relation entre le travail de mastication et certains éléments physiques ou chimiques de la plante mangée. 
g) Vérifier que s'il n'y a pas "rassasiement mécanique " (mastication) c'est le "rassasiement volumétrique " qui limite l'absorption des aliments, ou inversement.

\section{$2^{\circ}$ Travaux concernant les aliments fournis à la vache.}

a) Détermination du travail de mastication de nos différents aliments.

b) Influence du hachage et du déchiquetage sur ce travail.

c) Suivre l'évolution du travail de mastication nécessaire pour la même plante en fonction de certains facteurs : état végétatif au moment de la coupe, fréquence des coupes, variété de la plante, engrais apportés, place dans l'assolement, saison climatique, mode de récolte, etc...

L'étude de la méthode de récolte mérite à elle seule de nombreux travaux qui ne feront que s'ajouter à ceux déjà entrepris.

Ce qu'il faudrait surtout mieux considérer, c'est l'influence du mode de récolte sur le travail de mastication et le ballast. Jusqu'ici, on a surtout considéré l'influence du mode de récolte sur la teneur en protéines digestibles et unités amidon. Or, nous avons démontré ci-dessus que pour deux fourrages variant de $20 \%$ dans leur teneur en protéines digestibles, on trouvait que pour la même quantité de ballast absorbé, un des deux fourrages apportait trois fois plus de protéines que l'autre.

Il est possible que lorsqu'on ensile, deux acides paraissent donner deux ensilages de richesse voisine en protéines digestibles et unités amidon. Mais, si un des acides durcit la plante et qu'il faut deux fois plus de travail de mastication, on peut être certain que l'emploi de cet acide fera tomber le rendement en lait.

Si on sèche artificiellement un fourrage, on observera peut-être qu'une variation de Io $^{\circ}$ de la température de séchage ne change guère la teneur en protéines digestibles. Mais, si cette variation de température a doublé la teneur en ballast, c'est un grand malheur.

Après avoir exprimé ces désirs particuliers à nos savants et chercheurs, qu'il nous soit permis de leur exprimer un désir général : à savoir que dans leurs laboratoires et leurs centres de recherches, ils ne restent pas trop éloignés des cultivateurs et éleveurs. Les résultats de leurs recherches seront finalement jugés par ces éleveurs et leurs vaches.

\section{RESUME}

LEHMANN avait estimé qu'une vache était rassasiée quand elle avait absorbé une certaine quantité de ballast (matières organiques non digestibles de la ration). En général, on a admis en France et à l'étranger que la matière sèche de la ration représentait un meilleur critérium du rassasiement. FISSMER, à la suite d'essais remarquables, exprima la même opinion. 
En reprenant les essais et chiffres de FISSMER, j'ai essayé de prouver que la théorie de LEHMANn était exacte, mais que la quantité de basllat de 4,3 $\mathrm{kg}$ (pour une vache de $500 \mathrm{~kg}$ ) était un maximum jamais dépassé et que si cette quantité n'était pas atteinte, c'est qu'il y avait un "goulot " freinant le passage des aliments. Ce freinage provient du travail de mastication (compris dans un sens très général).

J'ai cru pouvoir déduire des essais de l'Université de Cornell que la vache ne pouvait fournir qu'un travail de mastication maximum déterminé et était incapable dę le dépasser, même si elle n'était pas rassasiée "volumétriquement " ou "physiologiquement ".

J'ai considéré qu'une vache était " rassasiée mécaniquement " quand elle avait accompli ce travail de mastication maximum. De même, je la considère " rassasiée volumétriquement" quand elle a absorbé la quantité maxima de ballast.

Suivant les aliments, c'est 1'un ou l'autre rassasiement qui limite la quantité maxima que peut manger la vache.

Me basant sur le coefficient W (coefficient d'utilisation) de KELLNER, je me suis efforcé de calculer le travail de mastication de certains aliments. Ces chiffres, comme cette théorie, doivent être vérifiés par des expériences pratiques.

Si cette théorie est exacte, nous espérons qu'elle permettra au cultivateur de pouvoir utiliser d'une manière plus pratique les tables d'alimentation, alors que jusqu'ici, le cultivateur a éprouvé beaucoup de difficultés à employer ces tables, et, à la vérité, ne les emploie guère.

Reçu pour publication le 15 Mars 1951 